\title{
FoxO1 and HNF-4 Are Involved in Regulation of Hepatic Glucokinase Gene Expression by Resveratrol*
}

Received for publication, July 29, 2009, and in revised form, September 2, 2009 Published, JBC Papers in Press, September 9, 2009, DOI 10.1074/jbc.M109.045260

Goutham Kumar Ganjam $^{\ddagger \S}$, Elitsa Y. Dimova ${ }^{\ddagger \rrbracket}$, Terry G. Unterman ${ }^{\| * * 1}$, and Thomas Kietzmann ${ }^{\ddagger \rrbracket 2}$

From the ${ }^{\ddagger}$ Faculty of Chemistry, Department of Biochemistry, University of Kaiserslautern, D-67663 Kaiserslautern, Germany, the

${ }^{\S}$ Department of Nutrient Physiology, Philipps University Marburg, Karl-von-Frisch-Strasse 8, D-35043 Marburg, Germany, the

"Department of Biochemistry, University of Oulu, P.O. Box 3000, FI-90014 Oulu, Finland, the "Departments of Medicine and

Physiology and Biophysics, University of Illinois College of Medicine at Chicago, Chicago, Illinois 60612, and the ** Jesse Brown

Veterans Affairs Medical Center, Chicago, Illinois 60612

Resveratrol, a polyphenol derived from grapes, exerts important effects on glucose and lipid metabolism, yet detailed mechanisms mediating these effects remain unknown. The liver plays a central role in energy homeostasis, and glucokinase (GK) is a key enzyme involved in glucose utilization. Resveratrol activates SIRT1 (sirtuin 1), which promotes deacetylation of the forkhead transcription factor FoxO1. Previously, we reported that FoxO1 can suppress and that HNF-4 can stimulate GK expression in the liver. Here, we examined the role of FoxO1 and HNF-4 in mediating resveratrol effects on liver GK expression. Resveratrol suppressed hepatic GK expression in vivo and in isolated hepatocytes, and knocking down FoxO1 with shRNAs disrupted this effect. Reporter gene, gel shift, supershift assay, and chromatin immunoprecipitation studies show that FoxO1 binds to the GK promoter and that the interplay between FoxO1 and HNF-4 within the GK promoter is essential for mediating the effects of resveratrol. Resveratrol promotes deacetylation of FoxO1 and enhances its recruitment to the FoxO-binding element. Conversely, resveratrol suppresses recruitment of HNF-4 to its binding site, and knockdown of FoxO1 blocks this effect of resveratrol. Coprecipitation and chromatin immunoprecipitation studies show that resveratrol enhances interaction between FoxO1 and HNF-4, reduces binding of HNF-4 to its own site, and promotes its recruitment to the FoxO site in a FoxO1-dependent manner. These results provide the first evidence that resveratrol represses GK expression via FoxO1 and that the interaction between FoxO1 and HNF-4 contributes to these effects of resveratrol.

The liver is a key organ in energy homeostasis, and glucokinase $(\mathrm{GK})^{3}$ plays a major role in promoting hepatic glucose utilization and maintenance of blood glucose homeostasis.

\footnotetext{
* This work was supported, in whole or in part, by National Institutes of Health Grant DK41430 (to T. G. U.). This work was also supported by the Department of Veterans Affairs Merit Review Program (to T. G. U.) as well as by grants from Fonds der Chemischen Industrie (to T. K.) and Deutsche Krebshilfe 106929 (to T. K.).

${ }^{1}$ To whom correspondence may be addressed: University of Illinois and Jesse Brown Veterans Affairs Medical Center, 820 S. Damen Ave., Chicago, IL 60612. Tel.: 312-569-7427; E-mail: unterman@uic.edu.

${ }^{2}$ To whom correspondence may be addressed: Dept. Biochemistry, University of Oulu, P.O. Box 3000, Fl-90014 Oulu, Finland. Tel.: 358-8-5537713; Fax: 358-8-5531141; E-mail: tkietzm@gwdg.de.

${ }^{3}$ The abbreviations used are: GK, glucokinase; shRNA, short hairpin RNA; ChIP, chromatin immunoprecipitation; FBEa and -b, FoxO-binding ele-
}

Compared with other hexokinases, GK has a smaller molecular mass (100 versus $52 \mathrm{kDa}$, respectively) and a lower affinity for glucose, with an $\mathrm{S}_{0.5}$ for glucose in the range of about 7-8 $\mathrm{mmol} /$ liter. Although GK binds to a regulatory protein (GKRP) and exists as a monomer, it displays sigmoidal kinetics with a Hill coefficient of about 1.5-1.7, indicating cooperativity with its substrate, glucose (1-3). These characteristics allow GK to react with glucose across the range of physiological glucose concentrations reached in vivo. Although GK is expressed predominantly in hepatocytes and pancreatic $\beta$-cells, it also is expressed in some neuroendocrine cells of the gastrointestinal tract and the brain, where it also may contribute to glucose sensing (4).

In the liver, GK is expressed predominantly in the less aerobic perivenous zone (5), and its expression is stimulated by insulin (6). Previous studies indicate that several transcription factors, including USF-1 and -2 (upstream stimulatory factor-1 and -2) $(7,8)$, HIF-1 (hypoxia-inducible factor-1) (9), PPAR $\gamma$ (peroxisome proliferator-activated receptor- $\gamma$ ) (10), sterol regulatory element binding protein-1c $(11,12)$, and hepatocyte nuclear factor-4 $\alpha$ (HNF-4 $\alpha)$ (13) may contribute to liver GK expression. We have reported that HNF-4 contributes to the effect of insulin on GK induction through a phosphatidylinositol 3' -kinase/protein kinase B-dependent mechanism (9). HNF-4 has been shown to interact with FoxO1 (14), an insulin-responsive transcription factor (15), suggesting the possibility that interactions between FoxO1 and HNF-4 may contribute to the regulation of GK expression.

FoxO transcription factors form a subgroup within the large family of Forkhead box (Fox) transcription factors (16), and they are important targets of insulin and growth factor action $(15,17)$. FoxO proteins promote the expression of genes that are adaptive to nutrient restriction and environmental stress $(15,17,18)$, and insulin signaling suppresses the effects of FoxO proteins on gene expression through signaling events mediated by the phosphatidylinositol 3'-kinase/protein kinase B pathway $(15,17,18)$. FoxO proteins contain threehighlyconserved consensus sites for phosphorylation by protein kinase B, corresponding to $\mathrm{Thr}^{24}, \mathrm{Ser}^{256}$, and $\mathrm{Ser}^{319}$ in human FoxO1 (19-22), and phosphorylation at

ment $a$ and $b$, respectively; $m F B E a$ and $-b$, mutant $F B E a$ and $b$, respectively; GM, Golgi membrane; IRE, insulin response element. 
these sites suppresses transactivation and promotes nuclear exclusion of FoxO proteins (22).

In the liver, FoxO1 has been shown to bind to insulin-responsive sequences and stimulate the expression of several genes involved in promoting hepatic glucose production, including phosphoenolpyruvate carboxykinase $(23,24)$ and glucose-6phosphatase $(25,26)$. Studies with transgenic mice expressing a constitutively active form of FoxO1 in the liver confirmed that FoxO1 stimulates the expression of glucose-6-phosphatase and phosphoenolpyruvate carboxykinase and indicated that FoxO1 proteins also reduce the expression of GK and several other genes involved in promoting glucose utilization (27). In addition, genetic studies in mice with targeted disruption of FoxO1 in the liver support the concept that FoxO1 is important for promoting both increased gluconeogenic gene expression and decreased GK expression when insulin signaling is disrupted $(28,29)$.

In addition to phosphatidylinositol 3 '-kinase and protein kinase $B$, several other signaling pathways and post-translational modifications contribute to the regulation of FoxO function (recently reviewed (30)), and considerable interest has focused on the role that acetylation plays in modulating the function of FoxO proteins (31-35). Acetylation of several lysine residues (corresponding to Lys ${ }^{242}$, Lys $^{245}$, and Lys ${ }^{262}$ in human FoxO1) located in the basic region at the $\mathrm{C}$-terminal end of the DNA binding domain of FoxO proteins is thought to impair DNA binding (36). SIRT1 (sirtuin 1), an $\mathrm{NAD}^{+}$-dependent deacetylase, has been shown to deacetylate these residues and modulate the function of FoxO proteins in mammalian cells (37).

Resveratrol, a polyphenol found in grapes, has been the subject of intense interest since it was shown to stimulate the function of SIRT1 and mimic sirtuin-dependent life span extension during caloric restriction in yeast (38). Animal studies indicate that resveratrol may have potential anticancer, anti-inflammatory, and neuro- and cardioprotective effects $(39,40)$ and can exert important effects on lipid and glucose metabolism, including effects on lipid accumulation, insulin sensitivity, blood glucose levels, and the number of mitochondria $(41-44)$. Studies in liver-derived rat H4IIE hepatoma cells indicate that resveratrol enhances gluconeogenic gene expression (33). Although resveratrol may exert effects on hepatic gene expression through deacetylation of FoxO1 (33) or other targets of SIRT1, including PGC-1 $\alpha$ (peroxisome proliferator receptor-coactivator-1 $\alpha$ ) (45), its role in regulating glycolytic gene expression (including $\mathrm{GK}$ ) and metabolism in the liver remains largely unknown.

Therefore, the aim of this study was to examine the effects of resveratrol on GK expression and activity in the liver and isolated hepatocytes. We find that treatment of rats and isolated primary hepatocytes with resveratrol suppresses GK expression, and knocking down the expression of FoxO1 with shRNAs disrupts this effect. Resveratrol treatment decreases the acetylation of FoxO1 in isolated hepatocytes, and reporter gene assays show that a modified form of FoxO1 (FoxO1-3KR) that mimics the effect of deacetylation has enhanced effects on GK promoter activity. Chromatin immunoprecipitation (ChIP) assays demonstrate that res- veratrol treatment enhances the recruitment of FoxO1 to the GK promoter, and reporter gene studies show that mutation of FoxO binding sites disrupts the effect of resveratrol on GK promoter activity. Further, co-precipitation studies, reporter gene assays, and ChIP analyses indicate that resveratrol promotes interaction between FoxO1 and HNF-4 and contributes to the repressive effects of resveratrol on GK expression within the context of the GK promoter. Together, these results provide new insight into mechanisms regulating hepatic GK expression and mediating effects of resveratrol and FoxO1 on GK expression in the liver.

\section{MATERIALS AND METHODS}

Animals-All studies were done in accordance with protocols approved by the Animal Use and Care Committee at the University of Kaiserlautern. Male Wistar rats (200-260 g) were kept on a 12-h day/night rhythm (light from 7 a.m. to 7 p.m.) with free access to water and a standard chow. Rats were treated with resveratrol by intraperitoneal injections of resveratrol dissolved in 30\% DMSO/saline at a dose of 5 or 10 $\mathrm{mg} / \mathrm{kg} /$ day for 2 days, and control animals were injected with $30 \%$ DMSO/saline alone. After 2 days, animals were anesthetized with pentobarbital (60 $\mathrm{mg} / \mathrm{kg}$ body weight) between 8 a.m. and 9 a.m., and blood was collected from the tail vein into a chilled syringe containing $10 \mathrm{IU}$ of heparin. Prior to preparation of hepatocytes, rats were anesthetized with pentobarbital ( $60 \mathrm{mg} / \mathrm{kg}$ body weight) between 8 a.m. and 9 a.m., as described (46).

Measurement of Plasma Glucose Levels-Animals were anesthetized by pentobarbital, and blood $(\sim 50 \mu \mathrm{l})$ was collected from the tail vein using a chilled syringe containing 10 IU heparin. The samples were centrifuged at full speed in a table top centrifuge for $3 \mathrm{~min}$, and $10 \mu \mathrm{l}$ of plasma was used for the determination of glucose with a glucose analyzer.

Plasmid Constructs-Luciferase (Luc) reporter gene plasmids containing the wild type rat GK promoter and the GK promoter mutated in the HNF binding site (13) or an array of five Gal4 binding sites upstream of the minimal E1b promoter upstream of the firefly luciferase cDNA (pG ${ }_{5} \mathrm{E} 1 \mathrm{~B}-\mathrm{Luc}$ ) (47) were described previously. The constructs pGL3rGK-mFBEa, pGL3rGK-mFBEb, and pGL3rGK-dmFBEab contain mutations of putative FoxO-binding element a or b (FBEa or FBEb) or both, respectively. They were generated with the primer 5'-GGG TTC AAG GCA ACT TAA GGA GAT TCT ATA ACA GTA AGC TTA AAA ATC TGA TTA AAA CGC-3' (a) or 5'-CTG ATT AAA ACG CAT CGT CGA CTA CTT GGG GTG GGG GGT GTC AGG GC-3' (b) and the wild type GK Luc construct as template by using the QuikChange XL sitedirected mutagenesis kit (Stratagene). Plasmids expressing p300 (48), rat HNF-4 $\alpha$ (49), or wild type FoxO1 have been described (21). The plasmids expressing the FLAG-tagged FoxO1-KR variant containing mutations replacing lysines 242, 245, and 262 with arginine, respectively, were a generous gift from Akiyoshi Fukamizu and have already been described (36).

Cell Transfection and Luciferase Assay-Rat hepatocytes were isolated by collagenase perfusion and maintained under standard conditions in an atmosphere of $16 \% \mathrm{O}_{2}, 79 \%$ 
$\mathrm{N}_{2}, 5 \% \mathrm{CO}_{2}(\mathrm{v} / \mathrm{v} / \mathrm{v})$ in medium M199 containing $0.25 \mathrm{nM}$ insulin added as a growth factor for culture maintenance; $100 \mathrm{~nm}$ dexamethasone, which is required as a permissive hormone; and, until the first change of medium after $5 \mathrm{~h}, 4 \%$ newborn calf serum, as previously described (50). Freshly isolated rat hepatocytes $\left(\sim 1 \times 10^{6}\right.$ cells/dish $)$ were transfected as described (50), and transfection efficiency was monitored by cotransfection with $0.25 \mu \mathrm{g}$ of Renilla luciferase expression vector (pRLSV40) (Promega). In brief, $2 \mu \mathrm{g}$ of the respective Luc construct was transfected with $500 \mathrm{ng}$ FoxO, HNF-4, and/or p300 expression vectors or with appropriate amounts of the respective empty expression vectors. For mRNA and protein analyses, $5 \mu \mathrm{g}$ of the respective transfection vector was used. After $5 \mathrm{~h}$, the medium was changed, and the cells were cultured for $19 \mathrm{~h}$; then the medium was changed again, and the cells were further cultured for $24 \mathrm{~h}(51)$.

Generation of FoxO1 shRNA-expressing Lentiviruses-Annealed FoxO1 shRNA oligonucleotides were cloned into the MluI and ClaI restriction sites of the vector PLVTHM (52). Two sequences for shRNA against FoxO1 were used. They are shRNA1 (5'-GCACCGACTTTATGAGCAA-3') and shRNA2 (5'-GGACAACAACAGTAAATTT-3'), respectively. The oligonucleotide with the sequence $5^{\prime}$-GCACGTTAAGTGCTACACA-3' was used to generate a scrambled shRNA control. Lentiviral particles expressing the respective shRNAs were generated by transfecting the three different plasmids into HEK $293 \mathrm{~T}$ cells. These plasmids are the pLVTHM vector carrying the oligonucleotides for shRNA, the pMD2G vector (53) encoding the envelope glycoprotein, and the second generation packaging plasmid psPAX2 (54). The expression of shRNA was verified in HEK cells, and the multiplicity of infection was determined by obtaining the optimal degree of target gene knockdown.

Infection of Primary Hepatocytes with an shRNA-expressing Lentivirus-Isolated rat hepatocytes were prepared as above, and after $5 \mathrm{~h}$, the fresh medium was replaced and infected with lentiviral vectors at a multiplicity of infection of about 40 for $14 \mathrm{~h}$. After $14 \mathrm{~h}$, the cells were washed twice with PBS, and fresh medium was given for up to $24 \mathrm{~h}$ depending on the experiment.

Western Blotting and Immunoprecipitation-Protein from primary cultured hepatocytes and transiently transfected hepatocytes was isolated as described (50). The protein content was determined using the Bradford method. $50 \mu \mathrm{g}$ of protein dissolved in $27 \mu \mathrm{l}$ of SDS sample buffer was loaded onto a $10 \%$ SDS-polyacrylamide gel and then transferred onto nitrocellulose membranes. Nonspecific binding was blocked with blocking buffer (10 mM Tris/ $\mathrm{HCl}$ ( $\mathrm{pH} 7.5), 100$ $\mathrm{mm} \mathrm{NaCl}, 0.1 \%$ Tween 20, $10 \%$ milk powder). Blots were incubated with primary goat antibody against GK (Santa Cruz Biochemistry, Heidelberg, Germany) in a 1:200 dilution. The rabbit polyclonal FoxO1 antibody (Santa Cruz, Heidelberg, Germany) and the rabbit polyclonal antibody against acetylated FoxO1 (amino acids $\mathrm{Lys}^{242} / \mathrm{Lys}^{245}$ ), kindly provided by Akiyoshi Fukamizu (36), were used in a 1:1000 dilution. Expression of tagged proteins was visualized with a monoclonal antibody against FLAG M2 (1:1000; Sigma) in blocking buffer overnight at $4{ }^{\circ} \mathrm{C}$. Washing was performed with blocking buffer without milk powder. The secondary antibodies were anti-goat IgG horseradish peroxidase (Dako, Hamburg, Germany), anti-rabbit IgG horseradish peroxidase, or anti-mouse IgG horseradish peroxidase (Santa Cruz Biotechnology) used in a 1:2000 dilution for $1 \mathrm{~h}$. The primary rabbit antibody against Golgi membrane (GM) (Bioscience, Göttingen, Germany) was used in a 1:8000 dilution. After washing for $30 \mathrm{~min}$, the ECL Western blotting system (Amersham Biosciences) was used for detection.

For co-immunoprecipitation experiments, cell lysates containing $150 \mu \mathrm{g}$ of protein were incubated with $2 \mu \mathrm{g}$ of antibody precoupled to protein G-Sepharose beads (Amersham Biosciences). Approximately $150 \mu \mathrm{l}$ of beads were washed twice with $1.5 \mathrm{ml}$ of lysis buffer ( $5 \times$ TBS, $10 \%$ Triton X-100, $0.5 \mathrm{M}$ EDTA, $0.5 \mathrm{M}$ EGTA, $125 \mathrm{~mm} \mathrm{Na}_{4} \mathrm{P}_{2} \mathrm{O}_{7}$ ) and centrifuged at $1000 \times g$ for $2 \mathrm{~min}$. Beads were resuspended in $500 \mu \mathrm{l}$ of lysis buffer with protease inhibitors, and $100 \mu \mathrm{l}$ were used for each pull-down reaction. Two $\mu \mathrm{g}$ of antibody were added to $150 \mu \mathrm{g}$ of whole cell extract and allowed to bind for $1 \mathrm{~h}$ at $4{ }^{\circ} \mathrm{C}$. After $1 \mathrm{~h}$, the samples were treated with washed beads and incubated overnight at $4{ }^{\circ} \mathrm{C}$ to pull down the interacting partner. The complexes bound to the beads were collected by centrifugation at $1000 \times g$ for 2 min and washed five times with lysis buffer. Washed beads were resuspended in SDS loading buffer, heated to $95^{\circ} \mathrm{C}$ for $7 \mathrm{~min}$, and resolved by SDS-PAGE and Western blot analysis.

RNA Preparation and Northern Analysis-Isolation of total RNA and Northern analysis were performed as described (13, 55). Digoxigenin-labeled antisense RNA hybridization probes were generated by in vitro transcription from $\mathrm{pBS}-\mathrm{GK}-1$ using T3 RNA polymerase or from pBS- $\beta$-actin using T7 RNA polymerase and RNA labeling mixture containing $3.5 \mathrm{~mm}$ 11-digoxigenin-UTP, $6.5 \mathrm{~mm}$ UTP, $10 \mathrm{~mm}$ GTP, $10 \mathrm{~mm} \mathrm{CTP,}$ and $10 \mathrm{~mm}$ ATP. Hybidizations and detections by chemiluminescence were carried out essentially as previously described (13). Blots were quantified with a videodensitometer (Biotech Fischer, Reiskirchen, Germany).

Chromatin Immunoprecipitation Assay-ChIP assays were performed as described $(56,57)$. Sonication of chromatin was titrated to prepare fragments of DNA, which was sheared to an average length of $\sim 200-300$ nucleotides, as determined by agarose gel electrophoresis and staining with ethidium bromide (not shown). Chromatin was precipitated overnight with either preimmune serum or $3 \mu \mathrm{g}$ of FoxO1 or HNF-4 antibodies, respectively, in a total volume of $1 \mathrm{ml}$ at $4{ }^{\circ} \mathrm{C}$. DNA from ChIP was analyzed by quantitative PCR using Taqman Gene Expression Master Mix (ABI). PCR was performed at $55^{\circ} \mathrm{C}$ for 35 cycles with primers that amplify a 342-bp region of the GK promoter containing the predicted FoxO binding sites (sense $(-720 /-702), 5^{\prime}$-TCCTGGCCAAACCCAAAG-3'; antisense (-396/-378), 5' -TTGGGTGGGAAGGAGAAG-3') or a nonoverlapping region of the proximal GK promoter that contains the HNF-4-binding element (sense (-263/-243), 5'-GGGTGGCTCCTGAAATACCC-3'; antisense $(-32 /-12), 5^{\prime}$-CCTCATGCCTACCTGTTTCG- $\left.3^{\prime}\right)$. The mRNA levels of $\beta$-actin were measured by PCR for normalization, and amplification of 
soluble chromatin prior to immunoprecipitation was used as an input control.

Preparation of Nuclear Extracts and Electrophoretic Mobility Shift Assay-Nuclear extracts were prepared as described previously (58). The sequences of GK oligonucleotides used for the electrophoretic mobility shift assay are as follows: FBEa, 5'-GATTCTATAACAGTAAAACAAAAATCTGAT3'; mutant FBEa (mFBEa), 5' -GATTCTATAACAGTAgcACAAcAATCTGAT-3'; FBEb, 5' -TTAAAACGCATATTGTTTTACTTGGGGTGGGG-3'; mFBEb, 5' -TTAAAACGCATATTGTgcTACTTGGGGTGGGG-3'. For supershift analysis, the nuclear extracts were preincubated on ice for $45 \mathrm{~min}$ with preimmune serum or FoxO1 antibody before adding the labeled probes.

GK Enzyme Activity-For GK enzyme activity, cells were washed with $0.9 \% \mathrm{NaCl}$; scraped in a buffer containing $50 \mathrm{~mm}$ Hepes, pH 7.5, $100 \mathrm{~mm} \mathrm{KCl,} 5$ mм $\mathrm{MgCl}_{2}, 1$ mм EDTA, $2.5 \mathrm{~mm}$ dithioerythritol, $10 \mathrm{mg} / \mathrm{ml}$ bovine serum albumin; and homogenized with an Ultraturrax (Jahnke and Kunkel, Stauffen). GK activity was then recorded essentially as previously described (13) and normalized to DNA as described (46).

\section{RESULTS}

Suppression of Glucokinase Expression by Resveratrol-To investigate the impact of resveratrol on GK expression in $v i v o$, rats were injected intraperitoneally with resveratrol. As shown in Fig. $1 A$, treatment with resveratrol for 2 days decreased GK mRNA levels in liver in a dose-dependent fashion (Fig. $1 A$ ), and this decrease in GK mRNA was accompanied by a reduction in GK protein levels (Fig. $1 A$ ). Conversely, resveratrol treatment enhanced expression of the gluconeogenic gene phosphoenolpyruvate carboxykinase (PEPCK) (Fig. 1B), consistent with previous studies indicating that it can enhance the expression of gluconeogenic genes in liver cells (33).

Next, to study the effect of resveratrol on GK expression at the cellular level, we performed studies in primary cultures of rat hepatocytes. As shown in Fig. $2 A$, treatment of primary rat hepatocytes with resveratrol suppressed the expression of GK in a dose-dependent fashion, and GK mRNA levels were decreased by $\sim 75 \%$ in hepatocytes treated with $50 \mu \mathrm{M}$ resveratrol. This decrease in mRNA was accompanied by a decrease in GK protein levels (Fig. 2A, bottom), similar to results in vivo. Conversely, treatment of hepatocytes with resveratrol enhanced phosphoenolpyruvate carboxykinase expression (Fig. 2A), consistent with the concept that resveratrol stimulates gluconeogenic gene expression (45).

We also measured GK enzyme activity in primary rat hepatocytes treated with resveratrol or, as a control, with insulin, which is known to stimulate GK expression (6). As shown in Fig. 2B, treatment with resveratrol reduced GK activity in a dose-dependent fashion in the presence of different glucose concentrations. In contrast, insulin induced GK enzyme activity $\sim 2$-fold, consistent with previous studies (13). As shown in Fig. 2C, Lineweaver-Burk analysis shows that treatment of cells with resveratrol or insulin does not change the $K_{m}$ of glucokinase for glucose (Fig. 2C). This result indicates that the effects of resveratrol on GK enzyme
(A)
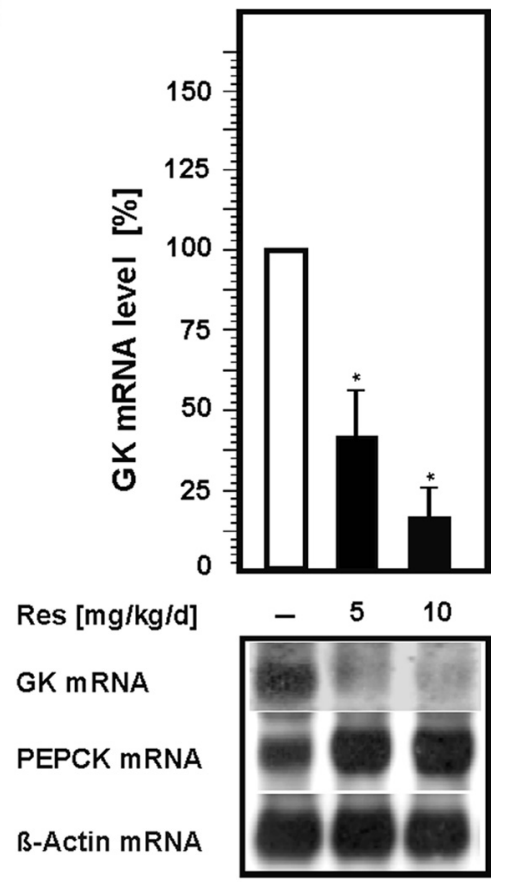

(B)

GK protein
B-Actin

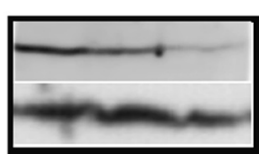

FIGURE 1. Reduction of GK expression in rat liver by resveratrol. $A$, Northern blot analysis. Total RNA was prepared from the livers of control and resveratrol treated rats. Then RNA from four animals in each group was pooled and analyzed by Northern Blotting with digoxigenin-labeled GK, phosphoenolpyruvate carboxykinase, and $\beta$-actin antisense RNA probes. Autoradiographic signals were detected by chemiluminescence. The graphs represent GK mRNA levels normalized for $\beta$-actin and indicate expression relative to control levels (100\%). Values are means \pm S.E. of three independent culture experiments. Statistical significance was determined by Student's $t$ test. ${ }^{*}$, significant difference, control versus resveratrol, $p \leq 0.05$. $B$, Western blot analysis. GK protein levels were analyzed by Western blotting, and the membrane was reprobed with a $\beta$-actin antibody as a loading control.

activity, including effects on $V_{\max }$ (Fig. $2 D$ ), are due primarily to changes in GK expression.

FoxO1 Is Critically Involved in Suppression of Glucokinase Expression and Promoter Activity by Resveratrol-Previous studies indicate that FoxO proteins are important targets of SIRT1 and resveratrol $(59,60)$. Since FoxO1 plays an important role in the regulation of hepatic gene expression, we asked whether knocking down FoxO1 levels would counteract the effect of resveratrol on GK expression and promoter activity in primary rat hepatocytes. Initial studies confirmed that lentiviruses expressing FoxO1 shRNA1 or FoxO1 shRNA2 (but not a scrambled shRNA) reduce protein levels of FoxO1 in rat hepatocytes by more than $80 \%$ (Fig. $3 A$ ).

As shown in Fig. 3B, FoxO1 shRNAs (but not scrambled shRNA) increased basal GK enzyme activity and blocked the ability of resveratrol to suppress GK enzyme activity in hepatocytes. This result indicates that FoxO1 plays a critical role in mediating the effect of resveratrol on GK in isolated hepatocytes.

As shown in Fig. 3C, resveratrol suppresses GK mRNA levels by about 75\%, and knocking down FoxO1 abolished 
(A)

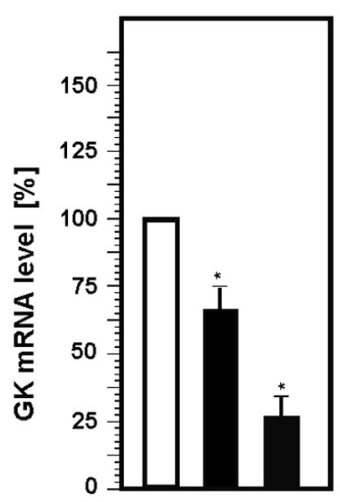

Resveratrol $[\mu \mathrm{M}] \quad-\quad 10 \quad 50$

GK mRNA

PEPCK MRNA

B-Actin mRNA

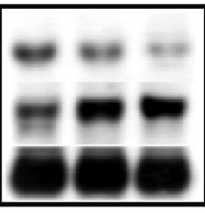

GK protein

B-Actin

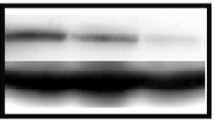

(B)

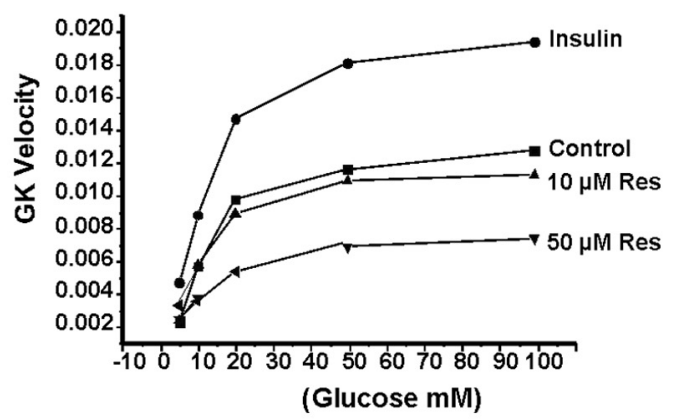

(C)

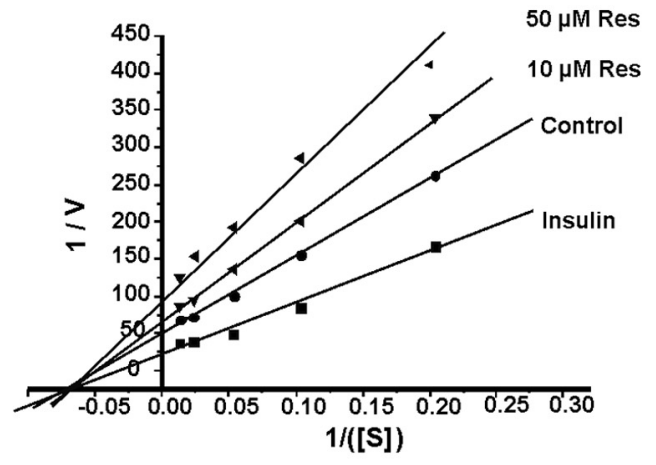

(D)

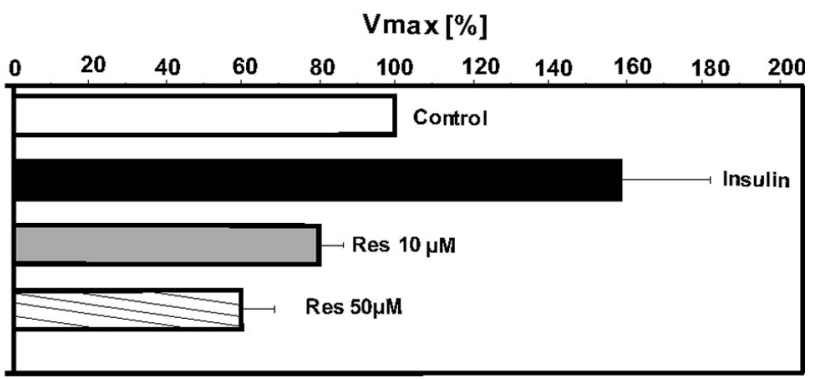

this effect, indicating that FoxO1 plays an important role in mediating effects of resveratrol on GK expression. Interestingly, in the absence of resveratrol, the FoxO shRNA increased the expression of GK mRNA above control, indicating that FoxO1 also contributes to the regulation of GK expression under basal conditions. Although resveratrol treatment also tended to reduce GK mRNA levels in cells treated with FoxO1 shRNA compared with shRNA alone, this effect was limited $(\sim 30 \%)$ and was not statistically significant. Together, these findings support the concept that FoxO1 plays an important role in mediating the effect of resveratrol on GK mRNA levels in hepatocytes.

To determine whether resveratrol (and FoxO1) suppresses the expression of GK at the level of transcription, we also performed luciferase reporter gene studies using constructs containing 1448 bp of the GK promoter. As shown in Fig. 3D, resveratrol treatment markedly suppressed activity of the GK promoter in primary hepatocytes. Again, FoxO1 shRNAs blocked this effect of resveratrol on GK promoter activity, demonstrating that FoxO1 is required for the effect of resveratrol on GK promoter activity in hepatocytes.

To determine whether FoxO1 exerts direct effects on GK expression, we also examined whether overexpression of FoxO1 alters the expression of GK mRNA and enzyme activity in primary rat hepatocytes. As shown in Fig. $4 A$, transfection with an expression vector for wild-type FoxO1 suppresses the level of GK mRNA in isolated hepatocytes compared with cells transfected with an empty vector. At the same time, FoxO1 stimulates the expression of phosphoenolpyruvate carboxykinase, consistent with previous studies (27). As shown in Fig. 4B, overexpression of FoxO1 also reduces GK enzyme activity in hepatocytes at different glucose concentrations. Kinetic analysis confirmed that the reduction of GK enzyme activity by FoxO1 is not due to a change in $K_{m}$ (Fig. 4C), indicating that the effect of FoxO1 on GK activity, including the effect on $V_{\max }$ (Fig. $4 D$ ), is due to changes in GK expression. Together, these results indicate that FoxO1 suppresses GK expression and is required for the ability of resveratrol to suppress GK activity and mRNA abundance at the level of gene expression in primary hepatocytes.

FIGURE 2. Repression of GK mRNA and GK enzyme activity by resveratrol in primary rat hepatocytes. Isolated hepatocytes were cultured for $24 \mathrm{~h}$; then medium was changed, and cells were treated with/without 10 or $50 \mu \mathrm{M}$ resveratrol or $100 \mathrm{~nm}$ insulin for $16 \mathrm{~h}$ prior to analysis of GK mRNA levels or GK activity, as indicated. $A$, Northern and Western blot analysis. $20 \mu \mathrm{g}$ of total RNA prepared from the cultured hepatocytes was analyzed by Northern blotting with digoxigenin-labeled GK, phosphoenolpyruvate carboxykinase, and $\beta$-actin antisense RNA probes. Autoradiographic signals were detected by chemiluminescence. The graphs represent GK mRNA levels normalized for $\beta$-actin and indicate expression relative to control levels (100\%). Values are means \pm S.E. of three independent culture experiments. Statistical significance was determined by Student's $t$ test. ${ }^{*}$, significant difference control versus resveratrol, $p \leq 0.05$. $B-D$, GK enzyme analysis. Glucokinase activity is presented as Michaelis-Menten $(B)$ and Lineweaver-Burk $(C)$ plots based on average results from three independent experiments. $D$, the $V_{\text {max }}$ for glucokinase enzyme activity calculated from three independent experiments for each condition. In each experiment, $V_{\text {max }}$ for each condition was expressed relative to the control (100\%). The $R^{2}$ values for the Lineweaver-Burk plots were 0.99 . 
(A)

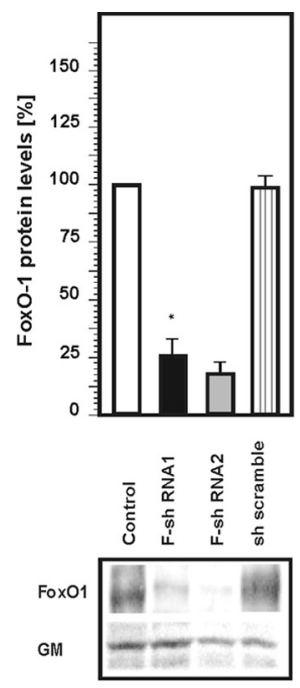

(B)

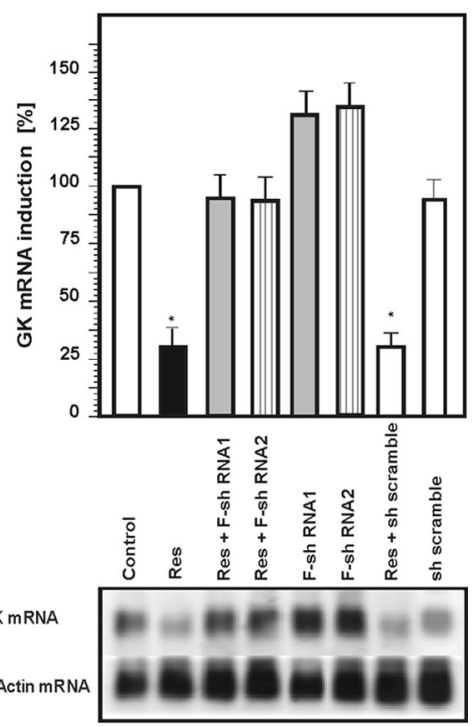

(C)

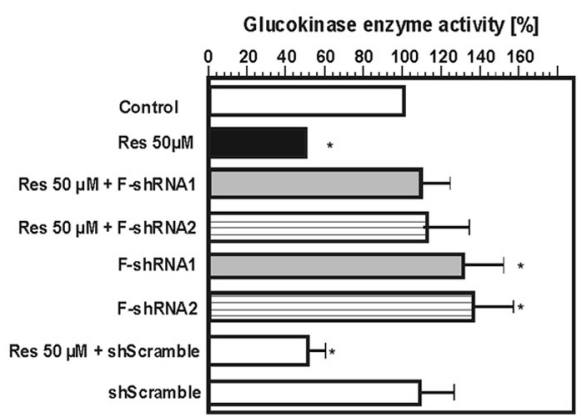

(D)

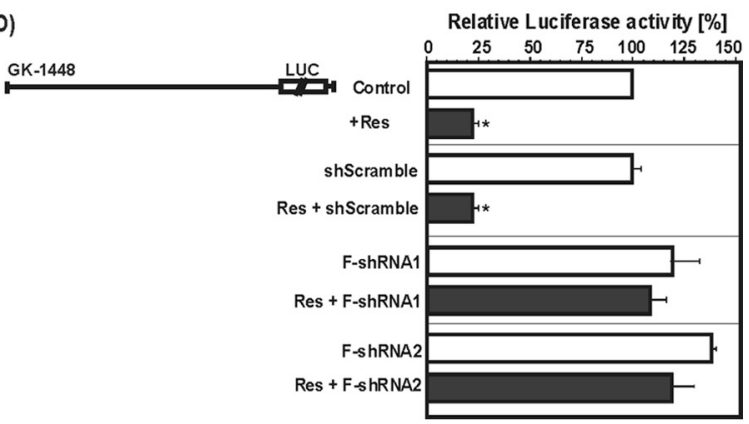

Resveratrol Promotes Deacetylation of FoxO1-Previous studies indicate that SIRT1 modulates the function of FoxO1 by promoting the deacetylation of lysine residues located within the basic region at the $\mathrm{C}$-terminal end of the FoxO1 DNA binding domain (36) and that resveratrol promotes SIRT1 deacetylase activity (61). To determine whether resveratrol promotes deacetylation of these sites in FoxO1 in primary hepatocytes, we examined the effect of resveratrol treatment on FoxO1 after it has been acetylated by a p300initiated mechanism (62). Cells were transfected with a FLAG-tagged FoxO1 expression vector with/without a vector expressing p300 and treated with/without resveratrol, and we examined the acetylation status of FoxO1 with an antibody specific for $\mathrm{Lys}^{242} / \mathrm{Lys}^{245}$-acetylated FoxO1 (62). As shown in Fig. $5 A$, the acetylation of FoxO1 is increased in cells co-transfected with the $\mathrm{p} 300$ expression vector, and this is reversed by treatment with $50 \mu \mathrm{M}$ resveratrol, indicating that resveratrol promotes the deacetylation of FoxO1 in hepatocytes.

To evaluate the role that changes in acetylation play in modulating the effects of FoxO1 on GK expression, we examined the effects of wild type FoxO1 and a modified form of FoxO1 in which lysine residues 242, 245, and 262 (which are subject to acetylation) are replaced by arginine residues (FoxO1-3KR). These lysine residues are located within the positively charged basic region at the C-terminal end of the FoxO DNA binding domain that is thought to be important for stabilizing DNA binding through electrostatic interactions. Acetylation of these residues is thought to reduce the positive charge in this region and impair binding to DNA target sites. Replacing these lysine residues with arginine residues prevents acetylation at these sites while maintaining the positive charge in this region of FoxO1.

To examine effects on FoxO1 function, we performed cotransfection studies using FoxO1 expression vectors and lucif-

FIGURE 3. Expression of Fox01 shRNA disrupts the effect of resveratrol on GK expression. $A$, knockdown of FoxO1. Primary hepatocytes were transduced with lentiviruses, allowing expression of FoxO1 shRNA1, FoxO1 shRNA2, or scrambled shRNA. FoxO1 protein levels were analyzed by Western blotting to show knockdown of FoxO1 protein levels by shRNAs. The membrane was reprobed with GM antibody as a loading control. The graphs represent FoxO1 levels normalized against GM and indicate expression compared with control levels (100\%). Values are means \pm S.E. of three independent culture experiments. *, significant difference, control versus shRNA,$p \leq 0.05$. $B$, Northern blot analysis. $20 \mu \mathrm{g}$ of total RNA prepared from cultured hepatocytes was analyzed by Northern blotting with digoxigeninlabeled GK and $\beta$-actin antisense RNA probes. Autoradiographic signals were detected by chemiluminescence. The graphs represent GK mRNA levels normalized for $\beta$-actin and indicate expression relative to control levels (100\%). Values are means \pm S.E. of three independent culture experiments. ${ }^{*}$, significant difference control versus resveratrol (Res) and control versus shRNA, $p \leq$ 0.05. C, effect of FoxO1 shRNA on GK enzyme activity. Primary hepatocytes were transduced with lentiviruses, allowing generation of FoxO1 shRNA1, FoxO1 shRNA2, or scrambled shRNA, and treated with resveratrol for $16 \mathrm{~h}$. GK enzyme activity was analyzed as in Fig. 1 . The $V_{\max }$ of the GK activity was calculated from three independent experiments for each condition. In each experiment, the $V_{\max }$ for each condition was expressed relative to the control (100\%). The $R^{2}$ values for the Lineweaver-Burk plots were 0.99. D, GK promoter activity. Cells were co-transfected with the $-1448 \mathrm{GK}$ promoter Luc reporter gene and Renilla Luc vectors and transduced with the viruses allowing generation of FoxO1 shRNA1 and FoxO1 shRNA2 or scrambled shRNA. Normalized Luc activity in the respective controls was set to $100 \%$. Values are means \pm S.E. of three independent experiments. ${ }^{*}$, significant difference, control versus resveratrol, $p \leq 0.05$. 
A) FoxO1

GK mRNA

PEPCK MRNA

B-Actin mRNA

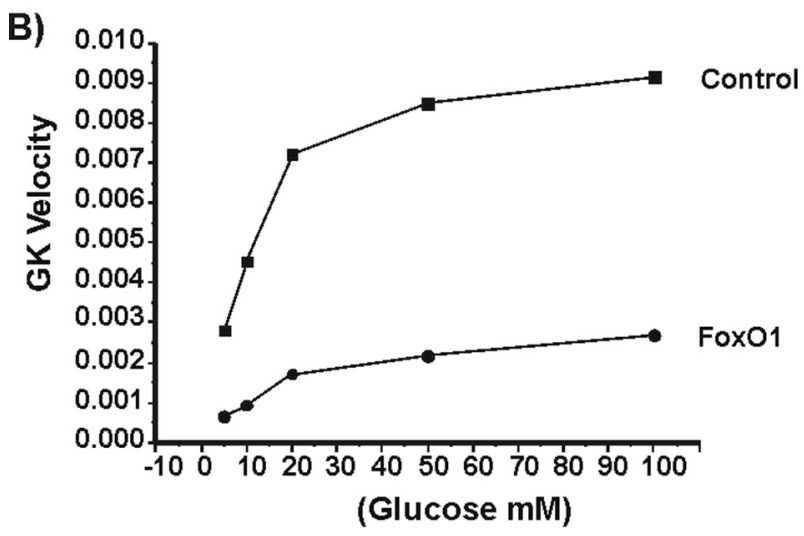

C)

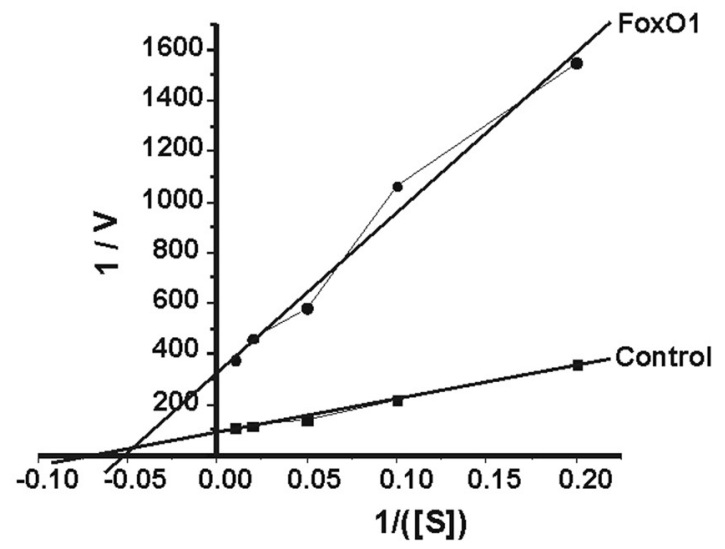

D)

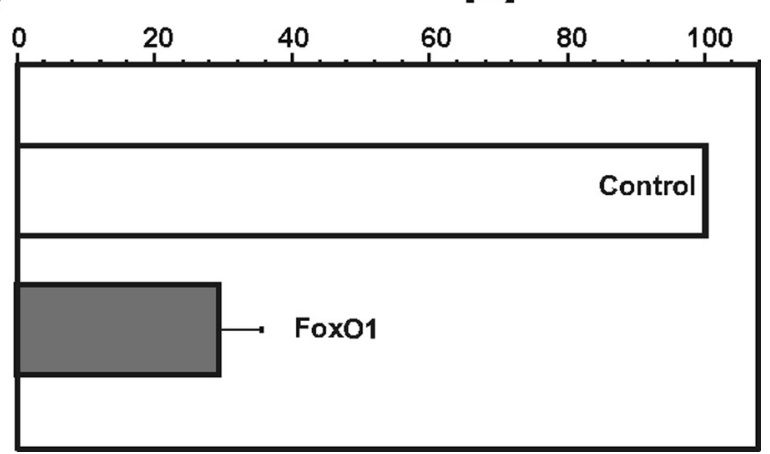

FIGURE 4. Repression of glucokinase mRNA and enzyme activity by Fox 01 in primary rat hepatocytes. $A$, effect on GK mRNA level. Isolated hepatocytes were transfected with FoxO1 expression vectors, and medium was changed $5 \mathrm{~h}$ after transfection. Medium was changed again $24 \mathrm{~h}$ later, and cells were cultured for an additional $24 \mathrm{~h}$ before analysis. $20 \mu \mathrm{g}$ of total RNA prepared from the cultured hepatocytes was analyzed by Northern blotting with digoxigenin-labeled GK and $\beta$-actin antisense RNA probes. Autoradiographic signals were detected by chemiluminescence. $B-D$, effect on GK enzyme activity. Hepatocytes were transfected with FoxO1 or empty vector as in $A$, and GK enzyme activity was measured as before. Effects on glucokinase activity are presented as Michaelis-Menten (B) and Lineweaver-Burk $(C)$ plots based on average results from three independent experiments. $D, V_{\max }$ of the glucokinase enzyme activity calculated from three independent experiments for each condition. In each experiment, $V_{\text {max }}$ for each condition was expressed relative to the control (100\%). The $R^{2}$ values for the Lineweaver-Burk plots were 0.99 . erase reporter gene constructs containing the liver-specific GK promoter $(-1448 \mathrm{GK}-\mathrm{Luc})$ or the insulin response element (IRE) from the glucose 6-phosphatase gene (IRE-thymidine kinase-Luc), which contains several FoxO binding sites and is known to respond positively to FoxO1 (63).

As shown in Fig. 5B, wild type FoxO1 decreased GK promoter activity by about $60 \%$, and this repressive effect was even more pronounced when the FoxO1-3KR mutant was expressed ( $80 \%$ decrease in promoter activity). In contrast, wild type FoxO1 increased luciferase activity in cells transfected with the IRE-thymidine kinase-Luc construct, consistent with previous studies (36), and this effect also was enhanced in cells expressing the FoxO1-3KR mutant (Fig. 4B). These results indicate that decreased acetylation of these residues enhances the function of FoxO1, including its ability to suppress the activity of the GK promoter.

FoxO Binding Sites Contribute to Effects of Resveratrol on GK Promoter Activity-To further explore the mechanisms by which FoxO1 and resveratrol modulate the function of the GK promoter, we considered whether FoxO1 may interact directly with the GK promoter and suppress promoter activity in hepatocytes. Based on a consensus sequence for FoxO binding sites ((C/G)(A/T)AAAA(C/T)AA) (15), we identified two putative FoxO1 binding sites $\left(5^{\prime}-{ }^{-567}\right.$ GTAAAACAA $^{-559}-3^{\prime}$ and $5^{\prime}$ - $^{-537}$ TTGTTTTAC ${ }^{-529}-3^{\prime}$ ) in the GK promoter, designated FBEa and -b, respectively. Next, we mutated these putative binding sites in the luciferase reporter gene construct containing the 1448-bp rat GK promoter individually (GK-1448 mFBEa and GK-1448 mFBEb) or together (GK-1448dmFBEab) and performed functional studies in primary hepatocytes. As shown in Fig. 6A, the ability of resveratrol to suppress GK promoter activity was partially reduced by mutation of FBEa (pGl3GK-1448 mFBEa) and was disrupted completely by mutation of FBEb (pGl3GK-1448 mFBEb) alone or by mutation of both FBEa and FBEb (pGl3GK-1448 mFBEab). These results indicate that FBEb is required for the full effect of FoxO1 to suppress GK promoter activity.

To examine the binding of FoxO1 to FBEa and/or FBEb, we performed electrophoretic mobility shift and supershift assays using double-stranded oligonucleotide probes containing either $\mathrm{FBEa}$ or $\mathrm{FBEb}$ or mutations of these sites (mFBEa or mFBEb, respectively). As shown in Fig. 6B, probes containing either FBEa or FBEb formed two rapidly migrating DNA protein complexes, as did probes containing mutations of the putative FoxO binding sites (mFBEa or mFBEb). Also, the formation of the two rapidly migrating complexes was not affected by the addition of either nonspecific IgG or antibody against FoxO1 (F-Ab). These results indicate that the formation of these complexes does not require the binding of FoxO1 to these probes.

In contrast, the FBEb probe also formed a third DNA protein complex with reduced mobility (labeled F), and the addition of a FoxO1 antibody (but not non-immune IgG) decreased the formation of this lower mobility complex formed with FBEb and resulted in formation of a supershift, indicating that this complex contains FoxO1. Together, these results indicate that FoxO1 interacts preferentially 
(A)

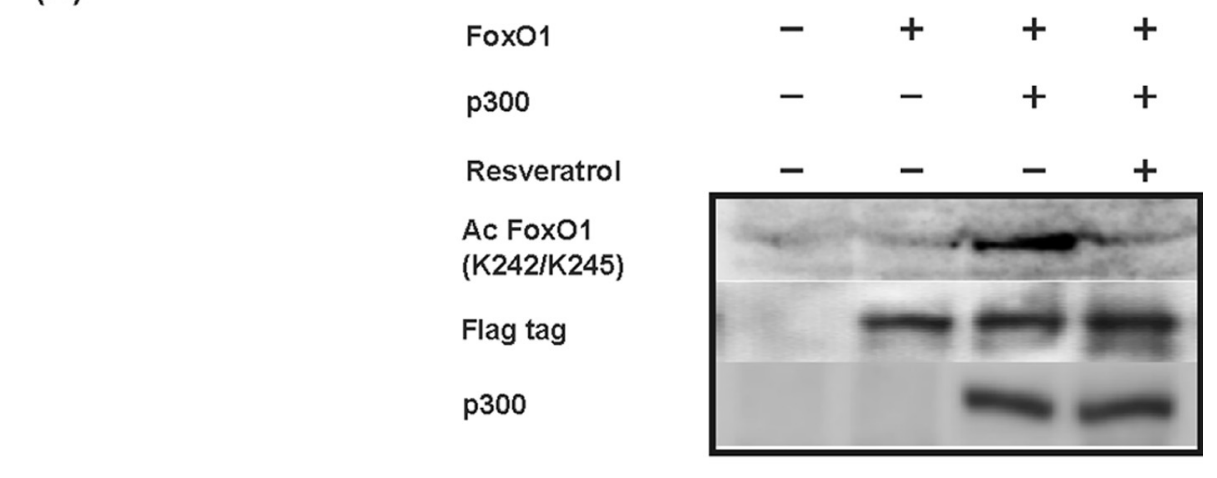

(B)

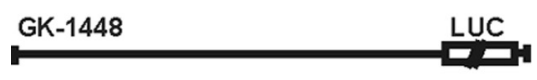

3XIRE-TK-Luc

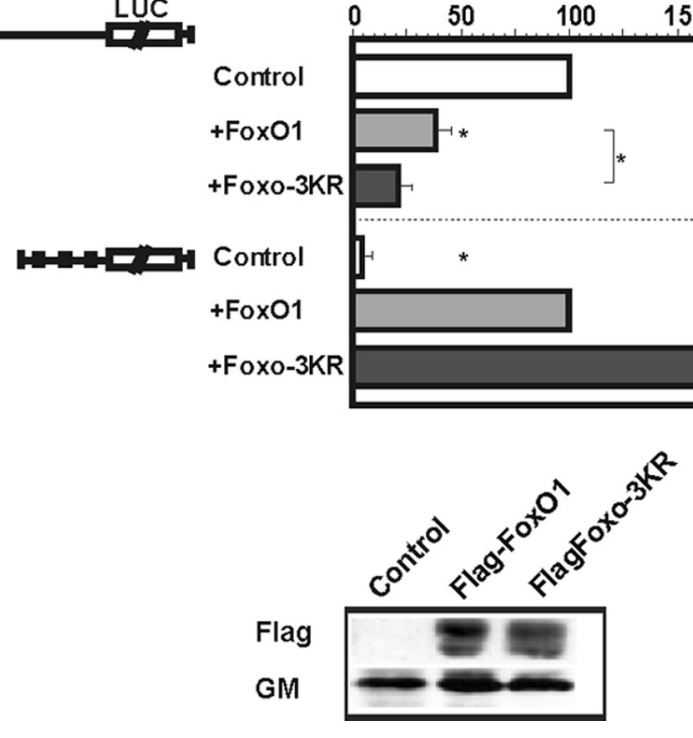

FIGURE 5. Resveratrol deacetylates Fox 01 and suppresses GK promoter activity. $A$, effect of resveratrol on FoxO1 acetylation. Primary hepatocytes were transfected with vectors expressing FLAG-tagged FoxO1 and/or p300 and were treated with/without $50 \mu \mathrm{m}$ resveratrol for $6 \mathrm{~h}$ as indicated. Acetylated FoxO1 in whole cell extracts $(150 \mu \mathrm{g})$ was detected by Western blotting using antibody specific for Lys $\mathrm{L}^{242} / \mathrm{Lys}^{245}$-acetylated FoxO 1 antibody, and p300 and FLAG-tagged FoxO1 were detected using anti-p300 and anti-FLAG antibody, respectively. The Golgi membrane protein detected by Western blotting was used to control for differences in loading. $B$, effect on GK promoter activity. Cells were co-transfected with expression vectors for either wild-type FLAG-FoxO1 or FLAG-FoxO1-3KR together with either the -1448GK promoter Luc reporter gene construct or a Luc reporter construct regulated by the FoxO1-binding IREs from the glucose-6-phosphatase promoter immediately upstream from the minimal thymidine kinase promoter (IRE-TK-LuC). Luciferase activity was expressed relative to the respective control (100\%). Values are means + S.E. of three independent experiments. *, significant difference, control versus resveratrol, $p \leq 0.05$. C, Western blot analysis. $50 \mu \mathrm{g}$ of protein from cells transfected with wild type FLAG-FoxO1 and mutated FLAG-FoxO1-3KR constructs were subjected to Western analysis with an antibody against FLAG tag or against GM. Autoradiographic signals were obtained by chemiluminescence.

with FBEb in the GK promoter, consistent with the results of reporter gene studies (above).

To determine whether endogenous FoxO1 interacts with the native GK promoter, we performed ChIP assays using nonspecific IgG or antibodies against FoxO1 and primers that amplify the region of the GK promoter containing FBEa and FBEb. As shown in Fig. 6C, PCR after immunoprecipitation with antibodies against FoxO1 confirmed that endogenous FoxO1 associates with this region of the GK promoter.

Next, we performed quantitative ChIP assays to determine whether resveratrol enhances the recruitment of FoxO1 to the GK promoter. As shown in Fig. $6 D$, quantitative PCR after immunoprecipitation with antibodies against FoxO1 demonstrates that overexpression of FoxO1 and treatment with resveratrol increases the association of FoxO1 with the GK promoter. In contrast, recruitment of FoxO1 to the GK promoter is reduced when cells are treated with insulin, consistent with previous studies indicating that insulin suppresses DNA binding and promotes the exclusion of FoxO1 from the nucleus.

HNF-4 Is Required for Resveratrol-and FoxO1-mediated Repression of GK Promoter Activity-We have previously reported that HNF-4 contributes to the regulation of GK expression in hepatocytes via a specific response element in the GK promoter (13), and FoxO1 has been reported to interact with HNF-4 (14). Accordingly, we asked whether interaction with HNF-4 may also contribute to effects of FoxO1 and resveratrol on GK expression. As shown in Fig. $6 A$, mutation of the HNF-4 binding site in the GK promoter (GK$1448 \mathrm{HNFm}$-Luc) disrupted the ability of resveratrol to suppress GK promoter activity, supporting the concept that HNF-4 is involved in mediating effects of resveratrol on GK expression.

To investigate in more detail whether FoxO1 and HNF-4 contribute to the regulation of GK, we performed co-transfection studies with FoxO1 and HNF-4 expression vectors in isolated hepatocytes. As shown in Fig. 7A, overexpression of HNF-4 stimulated GK mRNA levels, and co-expression of FoxO1 antagonized this effect of HNF-4, supporting the concept that FoxO1 antagonizes positive effects of HNF-4 on GK expression.

We next considered whether FoxO1 antagonizes the effect of HNF-4 on the activity of the GK promoter and whether FoxO binding sites are required for this effect. As shown in Fig. $7 B$, HNF-4 stimulates GK promoter activity by $\sim 3$-fold in isolated hepatocytes, consistent with our previous report (13). Cotransfection with an expression vector for FoxO1 impaired the effect of HNF-4 on GK promoter activity, demonstrating that FoxO1 antagonizes the ability of HNF-4 to stimulate the activity of the GK promoter. Mutation of FBEa did not disrupt the ability of FoxO1 to suppress basal or HNF-4-stimulated GK promoter activity. In contrast, mutation of FBEb abolished the ability of FoxO1 to suppress basal GK pro- 
(A)

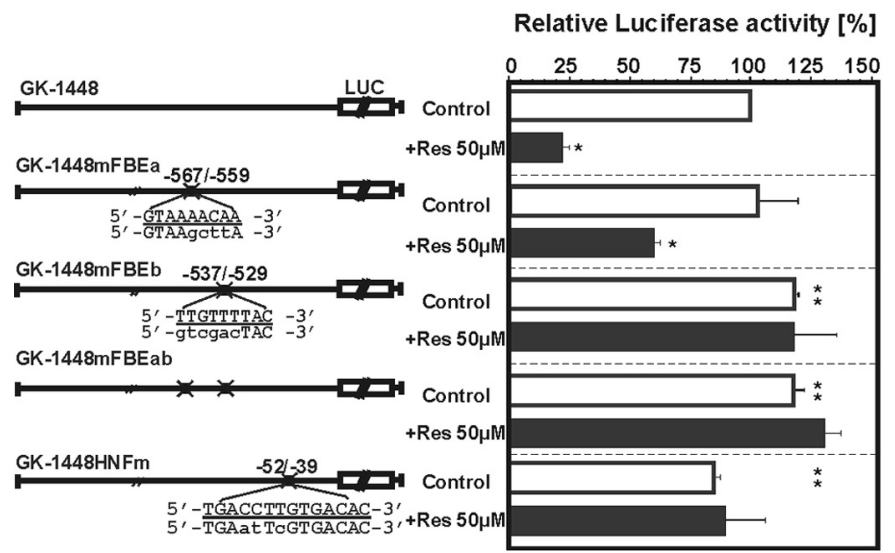

(B)

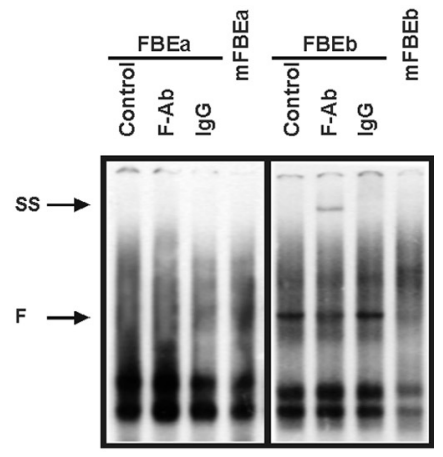

(C)

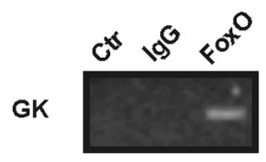

(D)

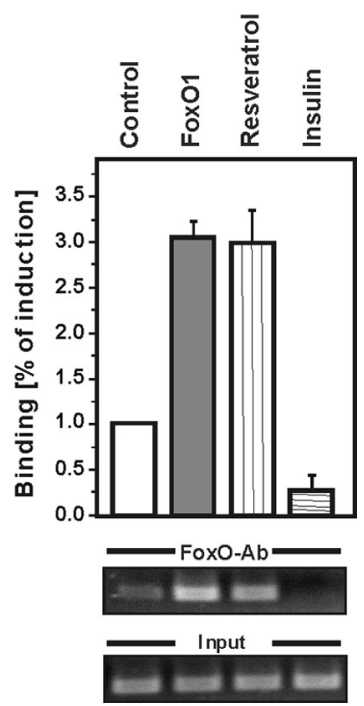

FIGURE 6. FoxO and HNF-4 binding sites are required for the effect of resveratrol on GK promoter activity. $A$, GK promoter activity. Primary rat hepatocytes were transiently transfected with luciferase reporter gene constructs containing either the wild type GK promoter extending $1448 \mathrm{bp}$ upstream from the transcription initiation site (GK-1448) or the 1448-bp promoter after it had been mutated either at FBEa (GK-1448 mFBEa), FBEb (GK$1448 \mathrm{mFBEb}$ ), FBEa and -b (GK-1448 dm FBEab), or the HNF-4 site located in the proximal promoter (GK-1448 mHNF4). Twenty-four h after transfection, the medium was changed, and cells were treated with $50 \mu \mathrm{m}$ resveratrol for $16 \mathrm{~h}$. In each experiment, luciferase activity is expressed relative to the appropriate GK-1448 Luc constructs (percentage of control). Values represent the moter activity. In addition, mutation of FBEb markedly antagonizes (but does not completely disrupt) the ability of FoxO1 to inhibit HNF-4-dependent stimulation of GK promoter activity (Fig. $7 B$ ). These results indicate that FBEb is important for the ability of FoxO1 to suppress both basal GK promoter function and to impair HNF-4-stimulated promoter activity. However, the latter also appears to involve a mechanism that does not require interaction with $\mathrm{FBEb}$.

As shown in Fig. 7B, mutation of the HNF-4 site disrupted the ability of HNF-4 to stimulate GK promoter activity but not the ability of FoxO1 to suppress basal promoter function. These results indicate that FoxO proteins suppress HNF-4-stimulated GK promoter activity largely by interfering with the ability of HNF-4 to stimulate promoter function through this binding site and that FoxO also can suppress basal GK promoter activity by other mechanisms.

Next, we asked whether resveratrol affects the interaction between FoxO1 and HNF-4 and/or their recruitment to the GK promoter. As shown in Fig. 8A, co-immunoprecipitation studies demonstrated that HNF-4 and FoxO1 proteins interact to form a complex in isolated hepatocytes that have been transfected with vectors expressing FoxO1 and HNF-4, consistent with previous studies (14). Interestingly, treatment of hepatocytes with resveratrol increased interaction between FoxO1 and HNF-4 (Fig. 8B), suggesting the possibility that enhanced interaction between FoxO1 and HNF-4 may contribute to effects of resveratrol on GK expression.

To address this question, we examined effects of resveratrol treatment on the recruitment of HNF-4 to the HNF-4 binding site, which we previously identified at $-52 /-39$ bp upstream from the transcription initiation site in the GK promoter (13). As shown in Fig. 8C, chromatin immunoprecipitation studies with an antibody against HNF-4 and primers that amplify the region between bp -262 and -12 confirm that HNF-4 interacts with this part of the GK promoter. As expected, overexpression of HNF-4 enhances the binding of HNF-4 to this site (Fig. 8D). In contrast, treatment with resveratrol or overexpression of FoxO1 markedly suppresses recruitment of HNF-4 to this region of the GK promoter, and knocking down FoxO1 with shRNA reverses the effect of resveratrol and increases the binding of HNF-4 above control levels (Fig. 8D). These

mean \pm S.E. of three independent experiments. ${ }^{*}$, significant differences between control and $50 \mu \mathrm{m}$ resveratrol, $p \leq 0.05$; **, significant differences between GK-1448 control and respective GK promoter mutant control, $p \leq$ 0.05 . $B$, electrophoretic mobility shift assay. The ${ }^{32}$ P-labeled GK-FBEa and FBEb and their respective mutant ( $\mathrm{mFBEa}$ and $\mathrm{mFBEb}$ ) double-stranded oligonucleotides probes were incubated with $10 \mu \mathrm{g}$ of protein of nuclear extracts. In electrophoretic mobility shift assays with antibody, the nuclear extracts were preincubated with either $2 \mu$ l of FoxO1 antibody $(F-A b)$ or nonimmune $\operatorname{lgG}$ for $2 \mathrm{~h}$ at $4{ }^{\circ} \mathrm{C}$ before adding the labeled probe. The DNA protein binding was analyzed by electrophoresis on $5 \%$ native polyacrylamide gels and phosphorimaging. SS, supershift. C, chromatin immunoprecipitation. Cells were treated with $50 \mu \mathrm{m}$ resveratrol or $100 \mathrm{~nm}$ insulin for $16 \mathrm{~h}$, and ChIP assays were performed using the FoxO1 (FoxO) or nonspecific IgG, and PCR was performed with primers amplifying the GK promoter containing the FBEa and FBEb, as outlined under "Materials and Methods." $D$, quantitative chromatin immunoprecipitation. Cells were treated with $50 \mu \mathrm{M}$ resveratrol for $16 \mathrm{~h}$. Quantitative ChIP assays were performed using the FoxO1 (FoxO1-Ab) antibody, and quantitative PCR was performed with primers amplifying the GK promoter containing FBEa and FBEb, as outlined under "Materials and Methods." Amplification of soluble chromatin prior to immunoprecipitation was used as an input control. 
results indicate that resveratrol and FoxO1 suppress the recruitment of HNF-4 to its binding site in the proximal GK promoter and that FoxO1 is required for this effect of resveratrol.

Since FoxO binding sites are important for the ability of both FoxO1 and resveratrol to suppress HNF-4-stimulated promoter activity, and resveratrol promotes interaction between FoxO1 and HNF-4, we next asked whether resveratrol treatment might promote the association of HNF-4 with FoxO1 that is bound to FoxO1 binding sites in the GK promoter. To address this question, we performed additional chromatin immunoprecipitation using the antibody against HNF-4 and primers that span the region of the GK promoter containing FBEa and FBEb (bp -702/-378) but not the HNF-4 binding site. As shown in Fig. $8 E$, chromatin immunoprecipitation studies demonstrate that HNF-4 also is associated with this region of the GK promoter, and this is increased by treatment with resveratrol. In addition, overexpression of FoxO1 also increases the recruitment of HNF-4 to this site, and knocking down FoxO1 with shRNA blocks the ability of resveratrol to promote the recruitment of $\mathrm{HNF}-4$ to this site. Taken together, these results indicate that resveratrol suppresses the recruitment of the HNF-4 to its own binding site in the proximal GK promoter and, at the same time, promotes its interaction with FoxO1 bound to the FoxO binding sites located further upstream.

\section{DISCUSSION}

In this study, we examined the effect of resveratrol on the expression of GK in vivo and in isolated hepatocytes and identified a novel mechanism by which resveratrol can suppress expression of the GK gene (i.e. by modulating the function of FoxO1 and its interaction with HNF-4). Previously, we reported that HNF-4 plays an important role in stimulating the
(A)

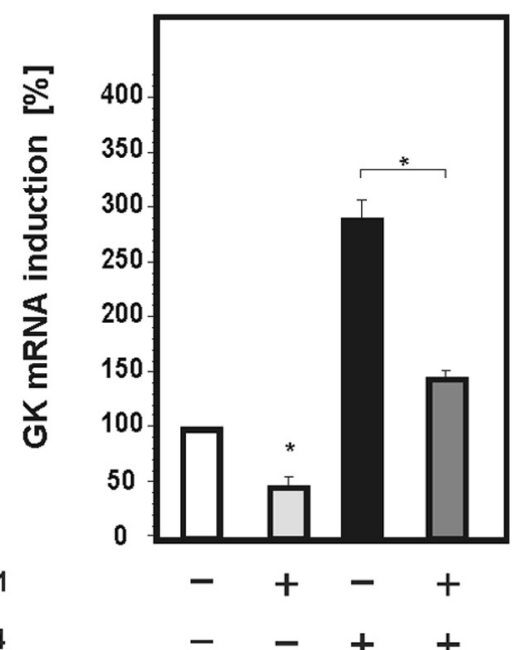

HNF-4

GK mRNA

ß-Actin mRNA

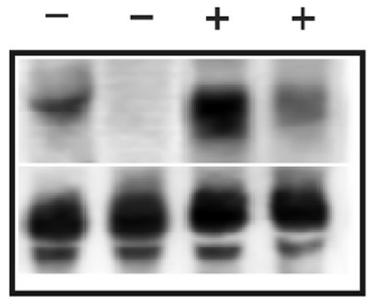

(B)

Relative Luciferase activity [\%]

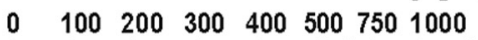

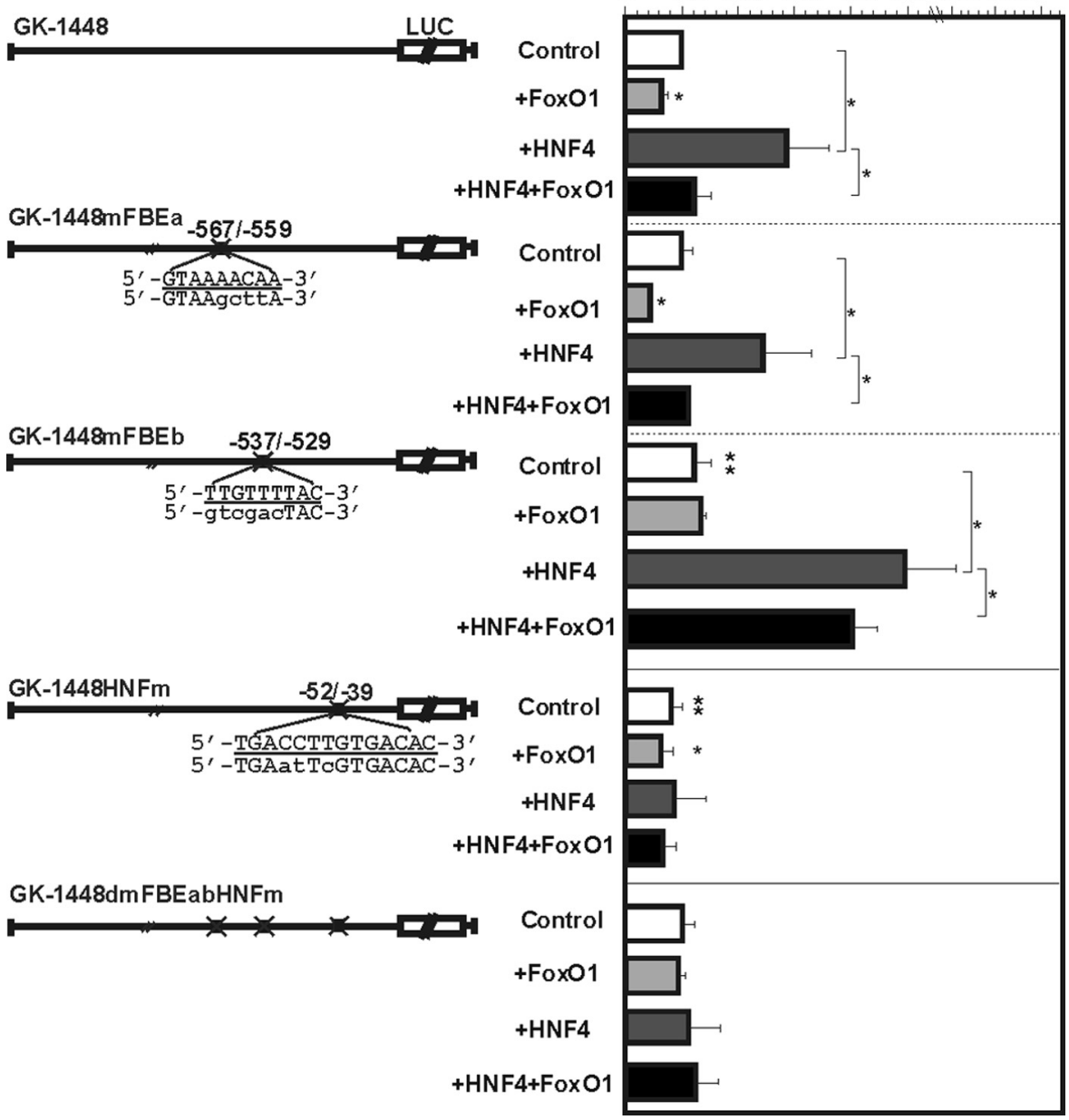


expression of GK in the liver and that an HNF-4 binding site in the proximal GK promoter is required for this effect (9). Previous studies also have shown that interaction between FoxO1 and HNF-4 disturbs HNF-4 function both by impairing its ability to bind to HNF-4 response elements (14) and by disrupting the function of the HNF-4 transactivation domain (64). The results of the present study indicate that resveratrol suppresses GK expression in the liver and that FoxO1 plays a critical role in this process, at least in part, by interfering with the recruitment and function of HNF-4 in the context of the GK promoter.

Our initial studies showed that resveratrol suppressed the expression of GK mRNA and that shRNA knockdown of FoxO1 disrupted this effect of resveratrol, indicating that FoxO1 plays a critical role. Analysis of the GK promoter sequence revealed the presence of $\mathrm{FBEa}$ and $\mathrm{FBEb}$, which are completely conserved in the rat and mouse GK promoters. The human GK promoter does not display conservation with FBEa, but FBEb shows conservation in 7 of 9 bases, including the AACAAA "core" sequence that is highly conserved in FoxO binding sites. These analyses together with gel shift and supershift studies and reporter gene studies identified $\mathrm{FBEb}$ as the major FoxO binding site contributing to the ability of resveratrol (and FoxO1) to suppress GK promoter activity. The HNF-4 binding site also is conserved in the mouse, rat, and human liver GK promoters. Interestingly, mutation of the HNF-4 binding site also disrupted the effect of resveratrol, suggesting that interaction between FoxO1 and HNF-4 may be important in mediating effects on GK promoter activity. Resveratrol promoted recruitment of FoxO1 to the region of the GK promoter and its interaction with HNF-4. At the same time, resveratrol suppressed association of HNF-4 with its binding site in the proximal GK promoter and enhanced its recruitment to the region of the promoter containing FoxO sites, and knocking down FoxO1 disrupted these effects of resveratrol. Taken together, these results indicate that FoxO1 plays a central role in mediating effects of resveratrol on hepatic GK expression and that interaction between FoxO1 and HNF-4 in the context of the GK promoter is critical for this effect.

The results of the present study also indicate that the ability of FoxO1 to suppress GK expression is potentiated upon resveratrol-mediated deacetylation of FoxO1 (65). Resveratrol, a polyphenol found in grapes and grape products, is among a number of small molecules that can stimulate the catalytic activity of SIRT1, an NAD-dependent deacetylase. Three lysine residues $\left(\mathrm{Lys}^{242}{ }^{24 y s^{245}}\right.$, and Lys ${ }^{262}$ ) located in the positively charged basic region of the DNA binding domain are acetylated when FoxO proteins interact with the p300 co-activator protein and SIRT1 targets the deacetylation of these residues (36). Using a site-specific antibody that detects acetylation of these residues in FoxO1, we demonstrated that resveratrol treatment of primary hepatocytes promotes deacetylation of FoxO1 at these residues. Using a mutant form of FoxO1 in which these acetylation sites were mutated, we also found that deacetylation enhances effects of FoxO1 on promoter activity, including its ability to suppress the activity of the GK promoter. Deacetylation of the FoxO1 residues maintains the positive charge in this region of the DNA binding domain and, thereby, enhances the stability of interactions between FoxO proteins and DNA target sites (36). Therefore, deacetylation at these residues may contribute to the ability of resveratrol to enhance the recruitment of FoxO1 to binding sites in the GK promoter in hepatocytes, as demonstrated in ChIP assays.

Previous studies regarding the role that acetylation plays in modulating the function of FoxO proteins have focused largely on its effect on interactions between FoxO proteins and their DNA binding sites. A recent paper published while this manuscript was in preparation reports that deacetylation of FoxO1 also potentiates the ability of FoxO1 to interact with and suppress the function of PPAR $\gamma(66)$. Based on our results, it is interesting to speculate that deacetylation also may promote interaction between FoxO1 and HNF-4 and thereby contribute to the suppression of GK expression. However, resveratrol also may promote interaction between FoxO1 and HNF-4 and the recruitment of FoxO1 to the GK promoter by other mechanisms, including effects on the activity of Akt (67).

Although FoxO proteins usually are thought to stimulate gene expression when they are recruited to a promoter (15), early studies suggested that they also can function as corepressor factors (68). Subsequent studies also indicated that the recruitment of FoxO1 to FoxO binding sites can suppress the function of some promoters, including the promoter for PDX-1 in $\beta$ cells (69), the agouti-related protein promoter in hypothalamic neurons (70), and the promoter for PPAR $\gamma$ in adipocytes (71). In the present study, our data indicate that FoxO1 inhibits GK expression in hepatocytes at least in part by interfering with the recruitment of HNF-4 to its binding site in the proximal GK promoter. This is consistent with previous studies indicating that FoxO1 can impair the ability of HNF-4 to bind to HNF-4 elements (14). We also found that treatment with resveratrol reduced the recruitment of HNF-4 to the HNF-4 site in a FoxO1-dependent manner,

FIGURE 7. Interaction of FoxO1 with HNF-4 contributes to FoxO-1-mediated GK repression. $A$, Northern blot analysis. Hepatocytes were transfected with FoxO1 or HNF-4 expression vectors, and medium was changed $5 \mathrm{~h}$ after transfection. Medium was changed again $24 \mathrm{~h}$ later, and cells were cultured for an additional $24 \mathrm{~h} .20 \mu \mathrm{g}$ of total RNA prepared from the cultured hepatocytes was analyzed by Northern blotting with digoxigenin-labeled GK and $\beta$-actin antisense RNA probes. Autoradiographic signals were detected by chemiluminescence. The graphs represent GK mRNA levels normalized against $\beta$-actin and indicate expression compared with control levels (100\%). Values are means \pm S.E. of three independent culture experiments. ${ }^{*}$, significant difference, $p \leq 0.05$, control versus FoxO1 or HNF-4 versus HNF-4 + FoxO1. B, effects of FoxO1 and HNF-4 on GK promoter activity. Hepatocytes were co-transfected with FoxO1 and/or HNF- $4 \alpha$ expression vectors and luciferase reporter gene constructs containing the wild-type GK-1448 promoter or the 1448-bp promoter mutated at the FBEa (GK-1448 mFBEa) site, the FBEb (GK-1448 mFBEb) site, the HNF-4 binding site (GK-1448 HNFm), or the FBEa, FBEb, and the HNF-4 sites (GK-1448 dmFBEabHNFm). In each experiment, the percentage of luciferase activity is expressed relative to the relevant GK-1448 Luc control construct (100\%). The values represent mean \pm S.E. of three independent experiments. *, significant differences, $p<$ 0.05 , control versus FoxO1, HNF- $4 \alpha$ or FoxO $1+\mathrm{HNF}-4 \alpha .{ }^{* *}$, significant difference between GK-1448 control and respective GK promoter mutant control; $p \leq 0.05$. 
(A)

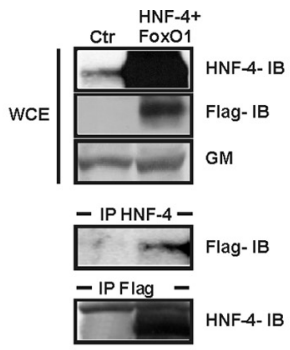

(B)

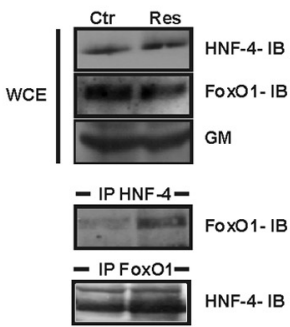

(C)

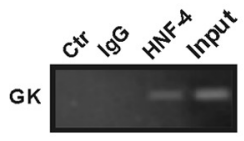

(D)
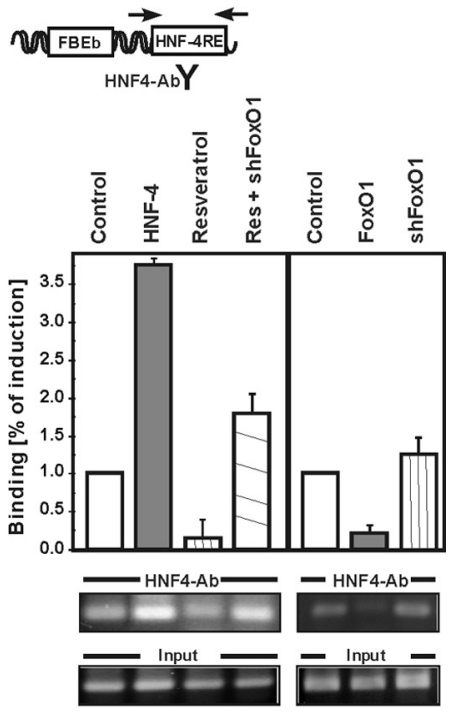

(E)

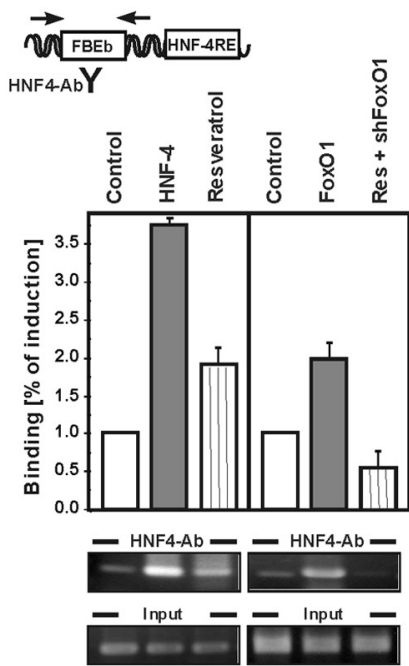

indicating that FoxO1 may mediate effects of resveratrol on GK expression through this mechanism.

Of note, FoxO1 also has recently been reported to suppress the expression of glucokinase in insulin-secreting $\beta$ cells (72), and this effect of FoxO1 (and the ability of IGF-I to stimulate the pancreatic glucokinase promoter) appears to be mediated through a cis-acting FoxO response element located in the upstream pancreatic GK promoter (73). Although the expression of glucokinase in the pancreas and the liver is directed by distinct tissue-specific promoters (74), these findings, taken together with the results of the present study, indicate that FoxO1 may contribute to the regulation of glucokinase gene expression in both the liver and pancreas through interaction with distinct cis-acting FoxO-responsive elements in the liver and pancreatic GK promoters.

Although the present study focused on the role that FoxO1 plays in mediating effects of resveratrol on GK expression, it is important to note that FoxO1 and its interaction with HNF-4 also may contribute to the regulation of GK in the liver by other factors, including insulin. Previous studies indicate that insulin plays an important role in regulating the expression of glucokinase in the liver $(75,76)$. FoxO1 stimulates the expression of gluconeogenic genes in the liver in fasting when nutrient availability and insulin levels are reduced, and inhibiting this effect of FoxO1 is important in mediating the ability of insulin to suppress hepatic glucose production and to control blood glucose levels (27, $77,78)$. We previously reported that the expression of constitutively active FoxO1 in the livers of transgenic mice not only stimulates the expression of gluconeogenic genes but also suppresses the expression of glucokinase (27). Recent studies using genetic models with targeted deletion of the FoxO1 gene in the liver also support the concept that FoxO1 can suppress GK expression $(28,29)$. Similarly, we have also

FIGURE 8. Resveratrol enhances interaction between FoxO1 and HNF-4 but decreases recruitment of HNF-4 to its binding site in the GK promoter. $A$, interaction between FoxO1 and HNF-4 in hepatocytes. Top, FoxO1, HNF-4, and GM levels were analyzed by Western blotting from hepatocytes with/without co-transfection of FoxO1 and HNF-4 expression vectors. Bottom, HNF-4-FoxO1 complexes were immunoprecipitated with anti-HNF-4 or FLAG antibody and were resolved by Western blotting with anti-FLAG or anti-HNF-4 antibody. $B$, resveratrol promotes FoxO1 and HNF-4 interaction in liver. Top, FoxO1, HNF-4, and GM levels were analyzed by Western blotting from liver extracts of rats treated with $10 \mathrm{mg} / \mathrm{kg}$ resveratrol for 2 days. Bottom, HNF-4-FoxO1 complexes were immunoprecipitated with either anti-HNF-4 or anti-FoxO1 antibody and were resolved by Western blotting with anti-HNF-4 or anti-FoxO1 antibody, respectively. $C$, chromatin immunoprecipitation. ChIP assays were performed using the HNF-4 or nonspecific $\operatorname{lgG}(I g G)$, and PCR was performed with primers amplifying the GK promoter containing the HNF-4 site as outlined under "Materials and Methods." $D$, quantitative chromatin immunoprecipitation. Cells were either transfected with HNF-4 expression vector or transduced with the viruses allowing generation of FoxO1 shRNA1 and left untreated or treated with $50 \mu \mathrm{m}$ resveratrol for $16 \mathrm{~h}$. Quantitative ChIP assays were performed using the HNF-4 (HNF-4-Ab) antibody, and quantitative PCR was performed as outlined under "Materials and Methods." Amplification of soluble chromatin prior to immunoprecipitation was used as an input control. E, chromatin immunoprecipitation. Quantitative ChIP assays were performed using the HNF-4 antibody, and quantitative PCR was performed with primers amplifying the GK promoter containing the FBEs but not the HNF-4 site, as outlined under "Materials and Methods." Amplification of soluble chromatin prior to immunoprecipitation was used as an input control. 


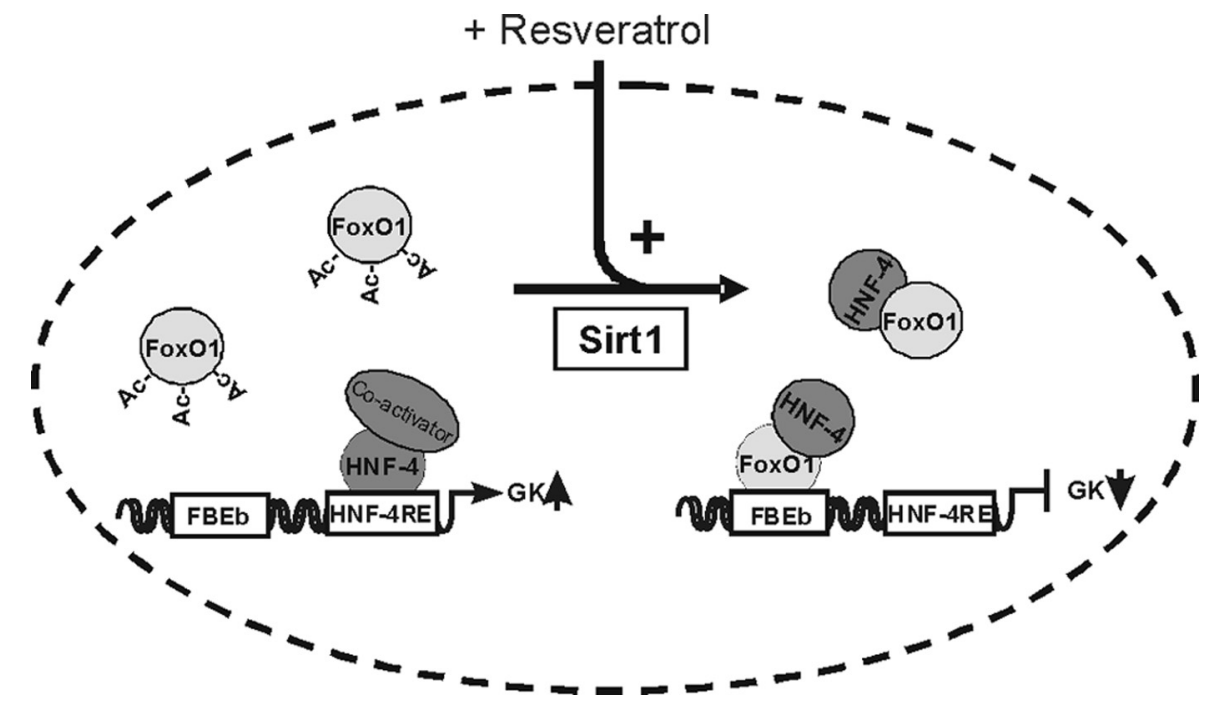

FIGURE 9. Model of GK repression in response to resveratrol and acetylation. Resveratrol promotes deacetylation of FoxO1, which can promote its binding to the GK FBEb and interaction of FoxO1 with HNF-4. Together, this enhances the ability of FoxO1 to displace HNF-4 away from its own binding site in the proximal promoter and recruit it onto the FoxO site through protein-protein interaction, where it is transcriptionally inactive due to its interaction with FoxO1. Other post-translational modifications, including phosphorylation/ dephosphorylation and interaction with 14-3-3 proteins, also may modulate the recruitment of FoxO1 to the GK promoter and its interaction with HNF-4 (not shown).

observed that expression of a constitutively active form of FoxO1 impairs the ability of insulin to stimulate glucokinase expression in primary cultures of hepatocytes. ${ }^{4}$ We previously identified an HNF-4 site located in the proximal GK promoter that is important for insulin regulation of GK promoter activity (9), and a recent report published while this paper was in preparation indicates that interaction of FoxO1 with HNF-4 is required for the regulation of GK and glucose6-phosphatase in response to insulin (79). Our present study extends these findings by additionally showing that not only the HNF-4 site but also FoxO sites and acetylation of FoxO1 play a role in regulation of GK expression. In addition, based on knockdown and ChIP experiments, our study shows that FoxO1 and resveratrol modulate recruitment of HNF-4 to the endogenous GK promoter. These findings, together with the results of the present study, indicate that interactions between FoxO1 and HNF-4 are important for the ability of both insulin and resveratrol to regulate the expression of GK and possibly other genes in the liver.

It is important to note that other transcription factors also have been implicated in the regulation of hepatic GK expression, including hypoxia-inducible factor-1 proteins (9), PPAR $\gamma$ (80), LXR (80), and sterol response element binding protein-1c (11). Interestingly, FoxO proteins can suppress the level of HIF- $1 \alpha$ at a post-transcriptional level (81) and also may suppress the expression of sterol regulatory element-binding protein-1c (27) and the function of PPAR $\gamma$ (82) and LXR (83). FoxO1 also can stimulate the expression of PGC-1 $\alpha$ (84), and PGC- $1 \alpha$ also has been reported to suppress glucokinase expression (85). Thus, FoxO1 may contribute to the regulation of GK expression through multiple mechanisms. This is consistent with our finding that the ability of FoxO1 to suppress basal GK

${ }^{4}$ G. Ganjam, T. Unterman, and T. Kietzmann, unpublished observations. promoter activity is not completely disrupted when the HNF-4 binding site in the proximal promoter is mutated. Nevertheless, the finding that mutation of the HNF-4 site disrupts the ability of resveratrol to suppress promoter activity and that knocking down FoxO1 with shRNAs also disrupts this effect of resveratrol strongly supports the concept that interaction between FoxO1 and HNF-4 is critical for mediating the effects of resveratrol on GK expression.

Based on these observations, we propose the following model by which resveratrol may reduce liverspecific GK expression (Fig. 9). Resveratrol enhances deacetylation of FoxO1, which may promote its recruitment to the GK promoter, where it can interact with HNF-4. At the same time, resveratrol also enhances interaction between FoxO1 and HNF-4, which impairs the binding of HNF-4 to its binding site in the proximal GK promoter. This interaction also promotes the recruitment of HNF-4 to the FoxO site. Since interaction with FoxO1 suppresses transactivation by HNF-4 (64), this results in suppression of GK promoter activity. Resveratrol also may promote recruitment of FoxO1 to the GK promoter and its interaction with HNF-4 by other mechanisms, including effects on the activity of Akt (67) (not shown).

Together, the results of this study extend our understanding of the mechanisms mediating effects of resveratrol on hepatic function and may provide insight into regulation of glucokinase under physiological conditions. In fasting, reduced action and increased activity of SIRT1 may both contribute to reduced expression of glucokinase, at least in part, by altering the function of FoxO1 and promoting its interaction with the GK promoter and HNF-4. Conversely, in the fed state, when insulin levels are high and the activity of SIRT1 is reduced, increased phosphorylation and acetylation of FoxO1 may serve in concert with HNF-4 to enhance glucose utilization in the liver by increasing the expression of glucokinase.

Acknowledgments-We gratefully acknowledge the helpful discussions with and suggestions of Dr. Patrick Iynedjian. We also thank Dr. Akitoshi Fukamizu for providing vectors and the antibody against acetylated FoxO1.

\section{REFERENCES}

1. Vandercammen, A., and Van Schaftingen, E. (1991) Eur. J. Biochem. 200, 545-551

2. Printz, R. L., Magnuson, M. A., and Granner, D. K. (1993) Annu. Rev. Nutr. 13, 463-496

3. Iynedjian, P. B. (1993) Biochem. J. 293, 1-13

4. Jetton, T. L., Liang, Y., Pettepher, C. C., Zimmerman, E. C., Cox, F. G., 
Horvath, K., Matschinsky, F. M., and Magnuson, M. A. (1994) J. Biol. Chem. 269, 3641-3654

5. Jungermann, K., and Kietzmann, T. (1996) Annu. Rev. Nutr. 16, 179-203

6. Iynedjian, P. B., Jotterand, D., Nouspikel, T., Asfari, M., and Pilot, P. R. (1989) J. Biol. Chem. 264, 21824-21829

7. Roth, U., Jungermann, K., and Kietzmann, T. (2004) Biol. Chem. 385, 239-247

8. Iynedjian, P. B. (1998) Biochem. J. 333, 705-712

9. Roth, U., Curth, K., Unterman, T. G., and Kietzmann, T. (2004) J. Biol. Chem. 279, 2623-2631

10. Kim, S. Y., Kim, H. I., Park, S. K., Im, S. S., Li, T., Cheon, H. G., and Ahn, Y. H. (2004) Diabetes 53, Suppl. 1, S66-S70

11. Foretz, M., Guichard, C., Ferré, P., and Foufelle, F. (1999) Proc. Natl. Acad. Sci. U.S.A. 96, 12737-12742

12. Kim, S. Y., Kim, H. I., Kim, T. H., Im, S. S., Park, S. K., Lee, I. K., Kim, K. S., and Ahn, Y. H. (2004) J. Biol. Chem. 279, 30823-30829

13. Roth, U., Jungermann, K., and Kietzmann, T. (2002) Biochem. J. 365, 223-228

14. Hirota, K., Daitoku, H., Matsuzaki, H., Araya, N., Yamagata, K., Asada, S., Sugaya, T., and Fukamizu, A. (2003) J. Biol. Chem. 278, 13056-13060

15. Barthel, A., Schmoll, D., and Unterman, T. G. (2005) Trends Endocrinol. Metab. 16, 183-189

16. Kaestner, K. H., Knochel, W., and Martinez, D. E. (2000) Genes Dev. 14, $142-146$

17. Greer, E. L., and Brunet, A. (2005) Oncogene 24, 7410-7425

18. Burgering, B. M., and Kops, G. J. (2002) Trends Biochem. Sci. 27, 352-360

19. Brunet, A., Bonni, A., Zigmond, M. J., Lin, M. Z., Juo, P., Hu, L. S., Anderson, M. J., Arden, K. C., Blenis, J., and Greenberg, M. E. (1999) Cell 96, $857-868$

20. Kops, G. J., de Ruiter, N. D., De Vries-Smits, A. M., Powell, D. R., Bos, J. L., and Burgering, B. M. (1999) Nature 398, 630-634

21. Guo, S., Rena, G., Cichy, S., He, X., Cohen, P., and Unterman, T. (1999) J. Biol. Chem. 274, 17184-17192

22. Zhao, X., Gan, L., Pan, H., Kan, D., Majeski, M., Adam, S. A., and Unterman, T. G. (2004) Biochem. J. 378, $839-849$

23. Yeagley, D., Guo, S., Unterman, T., and Quinn, P. G. (2001) J. Biol. Chem. 276, 33705-33710

24. Wolfrum, C., Asilmaz, E., Luca, E., Friedman, J. M., and Stoffel, M. (2004) Nature 432, 1027-1032

25. Schmoll, D., Walker, K. S., Alessi, D. R., Grempler, R., Burchell, A., Guo, S., Walther, R., and Unterman, T. G. (2000) J. Biol. Chem. 275, 36324-36333

26. Ayala, J. E., Streeper, R. S., Desgrosellier, J. S., Durham, S. K., Suwanichkul, A., Svitek, C. A., Goldman, J. K., Barr, F. G., Powell, D. R., and O’Brien, R. M. (1999) Diabetes 48, 1885-1889

27. Zhang, W., Patil, S., Chauhan, B., Guo, S., Powell, D. R., Le, J., Klotsas, A., Matika, R., Xiao, X., Franks, R., Heidenreich, K. A., Sajan, M. P., Farese, R. V., Stolz, D. B., Tso, P., Koo, S. H., Montminy, M., and Unterman, T. G. (2006) J. Biol. Chem. 281, 10105-10117

28. Matsumoto, M., Pocai, A., Rossetti, L., Depinho, R. A., and Accili, D. (2007) Cell Metab. 6, $208-216$

29. Dong, X. C., Copps, K. D., Guo, S., Li, Y., Kollipara, R., DePinho, R. A., and White, M. F. (2008) Cell Metab. 8, 65-76

30. Huang, H., and Tindall, D. J. (2007) J. Cell Sci. 120, 2479-2487

31. Accili, D., and Arden, K. C. (2004) Cell 117, 421-426

32. Daitoku, H., and Fukamizu, A. (2007) J. Biochem. 141, 769-774

33. Frescas, D., Valenti, L., and Accili, D. (2005) J. Biol. Chem. 280, 20589-20595

34. van der Horst, A., and Burgering, B. M. (2007) Nat. Rev. Mol. Cell. Biol. 8, $440-450$

35. Vogt, P. K., Jiang, H., and Aoki, M. (2005) Cell Cycle 4, 908-913

36. Matsuzaki, H., Daitoku, H., Hatta, M., Aoyama, H., Yoshimochi, K., and Fukamizu, A. (2005) Proc. Natl. Acad. Sci. U.S.A. 102, 11278-11283

37. Brunet, A., Sweeney, L. B., Sturgill, J. F., Chua, K. F., Greer, P. L., Lin, Y., Tran, H., Ross, S. E., Mostoslavsky, R., Cohen, H. Y., Hu, L. S., Cheng, H. L., Jedrychowski, M. P., Gygi, S. P., Sinclair, D. A., Alt, F. W., and Greenberg, M. E. (2004) Science 303, 2011-2015

38. Bordone, L., and Guarente, L. (2005) Nat. Rev. Mol. Cell. Biol. 6, 298-305

39. Harikumar, K. B., and Aggarwal, B. B. (2008) Cell Cycle 7, 1020-1035
40. Fulda, S., and Debatin, K. M. (2006) Cancer Detect. Prev. 30, 217-223

41. Kimura, Y., and Okuda, H. (2000) J. Pharm. Pharmacol. 52, 1287-1295

42. Cohen, H. Y., Miller, C., Bitterman, K. J., Wall, N. R., Hekking, B., Kessler, B., Howitz, K. T., Gorospe, M., de Cabo, R., and Sinclair, D. A. (2004) Science 305, 390-392

43. Heilbronn, L. K., Civitarese, A. E., Bogacka, I., Smith, S. R., Hulver, M., and Ravussin, E. (2005) Obes. Res. 13, 574-581

44. Nisoli, E., Tonello, C., Cardile, A., Cozzi, V., Bracale, R., Tedesco, L., Falcone, S., Valerio, A., Cantoni, O., Clementi, E., Moncada, S., and Carruba, M. O. (2005) Science 310, 314-317

45. Rodgers, J. T., and Puigserver, P. (2007) Proc. Natl. Acad. Sci. U.S.A. 104, $12861-12866$

46. Kietzmann, T. (2004) Methods Enzymol. 381, 357-376

47. Krüger, M., Schwaninger, M., Blume, R., Oetjen, E., and Knepel, W. (1997) Naunyn Schmiedebergs Arch. Pharmacol. 356, 433-440

48. Guo, S., Cichy, S. B., He, X., Yang, Q., Ragland, M., Ghosh, A. K., Johnson, P. F., and Unterman, T. G. (2001) J. Biol. Chem. 276, 8516-8523

49. Sladek, F. M. (1993) Receptor 3, 223-232

50. Immenschuh, S., Hinke, V., Ohlmann, A., Gifhorn-Katz, S., Katz, N., Jungermann, K., and Kietzmann, T. (1998) Biochem. J 334, 141-14.6

51. Liu, Q., Berchner-Pfannschmidt, U., Möller, U., Brecht, M., Wotzlaw, C., Acker, H., Jungermann, K., and Kietzmann, T. (2004) Proc. Natl. Acad. Sci. U.S.A. 101, 4302-4307

52. Wiznerowicz, M., and Trono, D. (2003) J. Virol. 77, 8957-8961

53. Colonno, R. J., and Banerjee, A. K. (1978) Cell 15, 93-101

54. Dull, T., Zufferey, R., Kelly, M., Mandel, R. J., Nguyen, M., Trono, D., and Naldini, L. (1998) J. Virol. 72, 8463-8471

55. Kietzmann, T., Roth, U., Freimann, S., and Jungermann, K. (1997) Biochem. J. 321, 17-20

56. Bonello, S., Zähringer, C., BelAiba, R. S., Djordjevic, T., Hess, J., Michiels, C., Kietzmann, T., and Görlach, A. (2007) Arterioscler. Thromb. Vasc. Biol. 27, 755-761

57. Klein, A., Flügel, D., and Kietzmann, T. (2008) Mol. Biol. Cell 19, 3667-3675

58. Dimova, E. Y., Möller, U., Herzig, S., Fink, T., Zachar, V., Ebbesen, P., and Kietzmann, T. (2005) Thromb. Haemost. 93, 1176-1184

59. Kim, D., Nguyen, M. D., Dobbin, M. M., Fischer, A., Sananbenesi, F., Rodgers, J. T., Delalle, I., Baur, J. A., Sui, G., Armour, S. M., Puigserver, P., Sinclair, D. A., and Tsai, L. H. (2007) EMBO J. 26, 3169-3179

60. Lagouge, M., Argmann, C., Gerhart-Hines, Z., Meziane, H., Lerin, C., Daussin, F., Messadeq, N., Milne, J., Lambert, P., Elliott, P., Geny, B., Laakso, M., Puigserver, P., and Auwerx, J. (2006) Cell 127, 1109-1122

61. Borra, M. T., Smith, B. C., and Denu, J. M. (2005) J. Biol. Chem. 280, 17187-17195

62. Fukuoka, M., Daitoku, H., Hatta, M., Matsuzaki, H., Umemura, S., and Fukamizu, A. (2003) Int. J. Mol. Med. 12, 503-508

63. Ghosh, A. K., Lacson, R., Liu, P., Cichy, S. B., Danilkovich, A., Guo, S., and Unterman, T. G. (2001) J. Biol. Chem. 276, 8507-8515

64. Li, T., Kong, X., Owsley, E., Ellis, E., Strom, S., and Chiang, J. Y. (2006) J. Biol. Chem. 281, 28745-28754

65. Daitoku, H., Hatta, M., Matsuzaki, H., Aratani, S., Ohshima, T., Miyagishi, M., Nakajima, T., and Fukamizu, A. (2004) Proc. Natl. Acad. Sci. U.S.A. 101, 10042-10047

66. Wang, F., and Tong, Q. (2009) Mol. Biol. Cell 20, 801-808

67. Zhang, J. (2006) Biochem. J. 397, 519-527

68. Zhao, H. H., Herrera, R. E., Coronado-Heinsohn, E., Yang, M. C., LudesMeyers, J. H., Seybold-Tilson, K. J., Nawaz, Z., Yee, D., Barr, F. G., Diab, S. G., Brown, P. H., Fuqua, S. A., and Osborne, C. K. (2001) J. Biol. Chem. 276, 27907-27912

69. Kitamura, T., Nakae, J., Kitamura, Y., Kido, Y., Biggs, W. H., 3rd, Wright, C. V., White, M. F., Arden, K. C., and Accili, D. (2002) J. Clin. Invest. 110, $1839-1847$

70. Kitamura, T., Feng, Y., Kitamura, Y. I., Chua, S. C., Jr., Xu, A. W., Barsh, G. S., Rossetti, L., and Accili, D. (2006) Nat. Med. 12, 534-540

71. Armoni, M., Harel, C., Karni, S., Chen, H., Bar-Yoseph, F., Ver, M. R., Quon, M. J., and Karnieli, E. (2006) J. Biol. Chem. 281, 19881-19891

72. Buteau, J., Shlien, A., Foisy, S., and Accili, D. (2007) J. Biol. Chem. 282, 287-293 


\section{Fox01 Regulates Glucokinase}

73. Yoshida, K., Murao, K., Imachi, H., Cao, W. M., Yu, X., Li, J., Ahmed, R. A., Kitanaka, N., Wong, N. C., Unterman, T. G., Magnuson, M. A., and Ishida, T. (2007) Endocrinology 148, 2904-2913

74. Magnuson, M. A. (1992) J. Cell. Biochem. 48, 115-121

75. Magnuson, M. A., Andreone, T. L., Printz, R. L., Koch, S., and Granner, D. K. (1989) Proc. Natl. Acad. Sci. U.S.A. 86, $4838-4842$

76. Iynedjian, P. B., Marie, S., Gjinovci, A., Genin, B., Deng, S. P., Buhler, L., Morel, P., and Mentha, G. (1995) J. Clin. Invest. 95, 1966-1973

77. Nakae, J., Kitamura, T., Silver, D. L., and Accili, D. (2001) J. Clin. Invest. 108, 1359-1367

78. Matsumoto, M., Han, S., Kitamura, T., and Accili, D. (2006) J. Clin. Invest. 116, 2464-2472

79. Hirota, K., Sakamaki, J., Ishida, J., Shimamoto, Y., Nishihara, S., Kodama, N., Ohta, K., Yamamoto, M., Tanimoto, K., and Fukamizu, A. (2008)
J. Biol. Chem. 283, 32432-32441

80. Kim, J. J., Li, P., Huntley, J., Chang, J. P., Arden, K. C., and Olefsky, J. M. (2009) Diabetes, in press

81. Tang, T. T., and Lasky, L. A. (2003) J. Biol. Chem. 278, 30125-30135

82. Fan, W., Imamura, T., Sonoda, N., Sears, D. D., Patsouris, D., Kim, J. J., and Olefsky, J. M. (2009) J. Biol. Chem. 284, 12188-12197

83. Kamei, Y., Miura, S., Suganami, T., Akaike, F., Kanai, S., Sugita, S., Katsumata, A., Aburatani, H., Unterman, T. G., Ezaki, O., and Ogawa, Y. (2008) Endocrinology 149, 2293-2305

84. Daitoku, H., Yamagata, K., Matsuzaki, H., Hatta, M., and Fukamizu, A. (2003) Diabetes 52, 642-649

85. Yoon, J. C., Puigserver, P., Chen, G., Donovan, J., Wu, Z., Rhee, J., Adelmant, G., Stafford, J., Kahn, C. R., Granner, D. K., Newgard, C. B., and Spiegelman, B. M. (2001) Nature 413, 131-138 


\section{FoxO1 and HNF-4 Are Involved in Regulation of Hepatic Glucokinase Gene Expression by Resveratrol}

Goutham Kumar Ganjam, Elitsa Y. Dimova, Terry G. Unterman and Thomas Kietzmann

J. Biol. Chem. 2009, 284:30783-30797.

doi: 10.1074/jbc.M109.045260 originally published online September 9, 2009

Access the most updated version of this article at doi: 10.1074/jbc.M109.045260

Alerts:

- When this article is cited

- When a correction for this article is posted

Click here to choose from all of JBC's e-mail alerts

This article cites 84 references, 45 of which can be accessed free at http://www.jbc.org/content/284/45/30783.full.html\#ref-list-1 